\title{
Estimating Transmitter Release Rates from Postsynaptic Current Fluctuations
}

\author{
Erwin Neher and Takeshi Sakaba \\ Max Planck Institute for Biophysical Chemistry, Department of Membrane Biophysics, D-37077 Göttingen, Germany
}

\begin{abstract}
A method is presented that allows one to estimate transmitter release rates from fluctuations of postsynaptic current records under conditions of stationary or slowly varying release. For experimental applications, we used the calyx of Held, a glutamatergic synapse, in which "residual current," i.e., current attributable to residual glutamate in the synaptic cleft, is present. For a characterization of synaptic transmission, several postsynaptic parameters, such as the mean amplitude of the miniature postsynaptic current and an apparent single channel conductance, have to be known. These were obtained by evaluating variance and two more higher moments of the current fluctuations. In agreement with Fesce et al. (1986), we found both by simulations and by analyzing experimental records that high-pass filtering of postsynaptic currents renders
\end{abstract}

Quantitative analysis of transmitter release is crucial for the understanding of mechanisms underlying various forms of plasticity, such as paired-pulse facilitation and short-term depression. However, estimation of release rates requires that the size and time course of the synaptic quantum [the miniature EPSC (mEPSC) for the case of excitatory transmission] are known. Furthermore, analysis of postsynaptic currents at glutamatergic synapses is complicated by the fact that the transmitter is cleared only slowly from the synaptic cleft, such that "residual glutamate" accumulates and activates "residual current" (Trussell et al., 1993; Barbour et al., 1994; Mennerick and Zorumski, 1995; Otis et al., 1996a; Kinney et al., 1997; Carter and Regehr, 2000). Neher and Sakaba (2001) adapted the method of deconvolution to allow for such residual current. By combining this method with the analysis of the variance of postsynaptic current records, they showed that the mEPSC amplitude decreases during prolonged stimulation attributable to desensitization of postsynaptic AMPA-type receptors. This confirmed the results of previous studies at various glutamatergic synapses, demonstrating both desensitization (Trussell et al., 1993; Otis et al., 1996b) and saturation (Clements et al., 1992; Jonas et al., 1993; Tang et al., 1994; Liu et al., 1999) of postsynaptic receptors during intense stimulation. Deconvolution was found to be very useful for studying large and rapid changes in transmitter release rate (Sakaba and Neher, 2001).

\footnotetext{
Received Aug. 23, 2001; revised Sept. 25, 2001; accepted Oct. 1, 2001.

This work was supported in part by a grant from the Deutsche Forschungsgemeinschaft (Sonderforschungsbereich 406) to E.N. and by an Alexander von Humbolt fellowship to T.S. We thank C. Pouzat for providing a macro for the detection of mEPSCs and L. de Felice, S. Pyott, R. Schnegenburger, and F. Sigworth for providing valuable advice regarding this manuscript.

Correspondence should be addressed to Erwin Neher, Max Planck Institute for Biophysical Chemistry, Department of Membrane Biophysics, D-37077 Göttingen, Germany. E-mail: eneher@gwdg.de.

Copyright (C) 2001 Society for Neuroscience $\quad 0270-6474 / 01 / 219638-17 \$ 15.00 / 0$
}

the estimates remarkably tolerant against nonstationarities. We also found that release rates and postsynaptic parameters can be reliably obtained when release rates are low $(\sim 10$ events/ $\mathrm{msec})$. Furthermore, during a long-lasting stimulus, the transmitter release at the calyx of Held was found to decay to a low, stationary rate of 10 events/msec after depletion of the "releasable pool" of synaptic vesicles. This stationary release rate is compatible with the expected rate of recruitment of new vesicles to the release-ready pool of vesicles. MiniatureEPSC (mEPSC) size is estimated to be similar to the value of spontaneously occurring mEPSC under this condition.

Key words: synaptic transmission; exocytosis; noise analysis; skewness; cumulants; calyx of Held; vesicle pool

However, the method is not very accurate during low, continuous release, in the presence of residual current. The analysis of EPSCs in such a situation is necessary not only at the calyx of Held but also at other synapses at which asynchronous release plays a major role in transmission (Parsons et al., 1994; Rieke and Schwartz, 1994; von Gersdorff and Matthews, 1994; Lu and Trussell, 2000). Therefore, it is desirable to explore an alternative method that is reliable under such conditions.

Fluctuation analysis has been used extensively in electrophysiology to study the properties of elementary events that underlie cellular signals, either channel currents or synaptic quanta (Katz and Miledi, 1972; Anderson and Stevens, 1973; Neher and Stevens, 1977; Colquhoun and Hawkes, 1981; Heinemann and Sigworth, 1993). However, the application of fluctuation analysis to the study of synaptic processes has been primarily restricted to either the statistical analysis of fluctuations in the amplitudes of evoked responses (for review, see Zucker et al., 1999; McLachlan, 1978) or the study of channel properties (Traynelis and Jaramillo, 1998). A few studies, however, have estimated release rates and mEPSC properties under stationary or slowly varying conditions (Segal et al., 1985; Fesce et al., 1986; Martin and Finger, 1988; Rossi et al., 1994). They demonstrated that such an analysis, based on an extension of Campbell's theorem, can be very powerful in elucidating mEPSC properties, particularly if high-passfiltered records are used.

Here, we adapt this method to the specific case of a large glutamatergic synapse, by calculating the variance and cumulants of higher order (skew and fourth cumulant) (Courtney, 1978; Segal et al., 1985; Fesce, 1990; Heinemann and Sigworth, 1991) of the postsynaptic current. By combining information from these quantities, we allow for the contribution of the residual current to noise and obtain reliable estimates for the amplitudes and rates of mEPSCs under moderately nonstationary conditions. In particu- 
lar, we estimate the release rate and mEPSC amplitude under the condition of deep depression (long-lasting strong stimulation) in which releasable vesicle pools should be empty and remaining release should be representing the delivery of new vesicles to the release-ready pool. We find a release rate that is compatible with the time course of recovery of the release-ready pool after stimulation, and we find normal mEPSC size. This latter finding indicates that, even after prolonged stimulation, partially filled vesicles do not contribute appreciably to the EPSC, as might be expected if vesicles recycle rapidly (Pyle et al., 2000). It also indicates that, even under these strong stimulation conditions, no vesicles contribute that are located more remote from postsynaptic densities.

\section{THEORY}

Following Segal et al. (1985) and Fesce (1990), we assume that the postsynaptic current is a sum of randomly occurring mEPSCs, each of which has a mean time course, $h \cdot F(t)$. Here, $h$ is an amplitude factor, and $F(t)$ is the time course of the mEPSC scaled to a peak amplitude of 1. Additionally, current fluctuations around the mean, attributable to the random opening and closing of channels, have to be considered. For simplicity, we assume that these fluctuations are independent of the mean mEPSC time course, have a Gaussian distribution, and are characterized by a variance, $V_{c}$, that is proportional to the total current. This assumption is problematic for the rising phase of the mEPSC, when channel openings are highly synchronized; however, for any given mEPSC, the variance attributable to the mean time course is large compared with the channel variance around its peak. The assumption is well warranted in the late decay phase of an mEPSC, when channels flicker is uncorrelated, and also for the residual current generated by slowly varying accumulations of neurotransmitter in the synaptic cleft. Below, we will demonstrate by simulation that the assumption is valid for the purpose of this study.

Campbell's theorem (Campbell, 1909; Rice, 1944), which is the basis of this technique, states that mean, variance, and the higher semiinvariants (or cumulants) of the noise signal generated by a stream of randomly occurring elementary events are proportional to the frequency of occurrence of elementary events, $\xi$, and to the integral over the $n$th power of the elementary signal waveform:

$$
\lambda_{n}=\xi h^{n} \int_{-x}^{+x}[F(t)]^{n} d t=\xi h^{n} I_{n} .
$$

Here $\lambda_{n}$ is the $n$th cumulant of the fluctuating signal, $\xi$ is the frequency of occurrence of elementary events, $h$ is the peak amplitude of the elementary signal (mEPSC, see above), and $I_{n}$ is a short-hand notation for the integral over the $n$th power of the normalized mEPSC time course $[F(t)]$. Three requirements have to be satisfied for Equation 1 to hold: (1) $\xi$, the rate of independently and randomly occurring mEPSCs, has to be reasonably constant within the observation interval, (2) $F(t)$ can be different from zero only within an interval short with respect to the observation interval, and (3) the total current is a linear superposition of randomly occurring elementary events (Rice, 1944; Segal et al., 1985).

When these conditions are satisfied, Equation 1, applied to the cases $n=2$ (variance) and $n=1$ (mean), readily leads to the equation used for standard noise analysis $\left(h=\lambda_{2} / \lambda_{1} \cdot\left(I_{1} / I_{2}\right)\right)$. However, in the presence of a residual current, standard noise analysis is not applicable, because the elementary event is very long lasting (including a long "tail" of its contribution to the residual current) and the ratio $I_{2} / I_{1}$ cannot be evaluated because of the ill-defined and variable nature of the residual current. Also, residual current is most likely a nonlinear function of residual glutamate, which violates the assumption of linear superposition. However, a high-pass-filtered version of the original current more readily satisfies the requirement of Campbell's theorem because high-pass filtering turns the elementary events into short, spike-like signals and eliminates the slowly-varying nonlinear components. Additionally, highpass filtering allows more accurate measurement of the skew and higher moments (Segal et al., 1985). After high-pass filtering, however, the mean of the signal is zero and can no longer be used for calculation of $\xi$. For the case that the variance originating from channel flickering, $V_{c}$, is small after high-pass filtering, an estimate for $\xi$ can be obtained from the ratio of skew and variance instead. Unfortunately, we found that, at the calyx of Held, channel variance, $V_{c}$, very often makes a substantial contribution to the total variance, especially when cyclothiazide (CTZ) is used to block glutamate receptor desensitization (Trussell et al., 1993; Yamada and Tang, 1993). However, $V_{c}$ is expected to be proportional to the mean current (as measured before high-pass filtering). If, as argued above, $V_{c}$ is statistically independent of mEPSC variance $\lambda_{2}^{\prime}$, the total variance after filtering can be written as a sum of the two contributions:

$$
\lambda_{2}^{\prime}=\xi h^{2} I_{2}^{\prime}+i^{\prime} I_{p}
$$

where $i^{\prime}$ is a proportionality constant representing the filtered single channel current, $I_{2}^{\prime}$ is the integral over the square of the filtered EPSC time course, and $I_{p}$ is the mean postsynaptic current before high-pass filtering. The primes $(')$ in this and the following equations denote the fact that they refer to the filtered record.

The skew $\left(\lambda_{3}^{\prime}\right)$ of the filtered postsynaptic current does not contain contributions from the residual current (the skew of a Gaussian distribution is zero) and is, therefore, given by the following:

$$
\lambda_{3}^{\prime}=\xi h^{3} I_{3}^{\prime},
$$

where $I_{3}^{\prime}$ is the integral of the filtered mEPSC raised to the third power.

From Equations 2 and 3, we obtain the following:

$$
h=\frac{\lambda_{3}^{\prime} I_{2}^{\prime}}{\left(\lambda_{2}^{\prime}-i^{\prime} I_{p}\right) I_{3}^{\prime}}
$$

and

$$
\xi=\frac{\left(\lambda_{2}^{\prime}-i^{\prime} I_{p}\right)^{3} \cdot I_{3}^{\prime 2}}{\lambda_{3}^{\prime 2} \cdot I_{2}^{\prime 3}}
$$

Because $\lambda_{2}^{\prime}, \lambda_{3}^{\prime}$, and $I_{p}$ can readily be measured and both $I_{2}^{\prime}$ and $I_{3}^{\prime}$ can be calculated from the mEPSC time course (see legend of Fig. 1), these equations allow the amplitude, $h$, and the rate, $\xi$, of mEPSCs to be calculated from postsynaptic current traces. $i^{\prime}$, the proportionality constant between channel variance and mean current, has to be determined independently as described in Results. Alternatively, it can be calculated from the cumulants (including information from the fourth cumulant, $\lambda_{4}$; see below). The fourth cumulant $\lambda_{4}$ is given by the following:

$$
\lambda_{4}=\mu_{4}-3 \lambda_{2}=\xi h^{4} I_{4},
$$

where $\mu_{4}$ is the fourth central moment. Like the other cumulants, $\lambda_{4}$ has the property that, when derived from a sum of two statistically independent signals, it is equal to the sum of the two cumulants. Furthermore, both the skew and the fourth cumulant of a random signal with Gaussian distribution are zero, such that the channel variance does not contribute to either of them. We therefore obtain, in analogy to Equations 4 and 5, the following:

$$
h=\frac{\lambda_{4}^{\prime}}{\lambda_{3}^{\prime}} \frac{I_{3}^{\prime}}{I_{4}^{\prime}}
$$

and

$$
\xi=\frac{\lambda_{3}^{\prime 4}}{\lambda_{4}^{\prime 3}} \frac{I_{4}^{\prime 3}}{I_{3}^{\prime 4}} .
$$

In practice, however, $\lambda_{4}^{\prime}$ can be determined with reasonable accuracy only for long stretches of data; therefore, as a rule, we determine $h$ and $\xi$ from Equations 4 and 5.

In some of our records, however, we encounter suitable stretches of data with relatively large residual current and little mEPSC activity. From these, $i^{\prime}$ can be calculated, because the variance is dominated by residual current. mEPSC-induced fluctuations, in this case, constitute a minor contribution, which can be taken into account by combining Equations 2, 3, and 6 for the calculation of $i^{\prime}$ :

$$
i^{\prime}=\frac{\lambda_{2}^{\prime}}{I_{p}} \cdot\left(1-\frac{\lambda_{3}^{\prime 2}}{\lambda_{2}^{\prime} \lambda_{4}^{\prime}} \frac{I_{2}^{\prime} I_{4}^{\prime}}{I_{3}^{\prime 2}}\right)
$$

It can be seen that $i^{\prime}$ is given by the ratio $\lambda_{2}^{\prime} / I_{p}$ (which is the expression for pure channel-derived fluctuations) and a correction term involving the ratio of third and fourth cumulants. We thus can calculate $i^{\prime}$ on a suitable segment and determine $h$ and $\xi$ according to Equations 4 and 5 for the rest of the trace unless there is reason to believe that $i^{\prime}$ varies 
along that trace. It will be shown below that $h$ can be calculated at much better resolution than $\xi$. Thus, it is advantageous to calculate $h$ from Equation 4 and insert this (averaged over longer stretches of data) together with $i^{\prime}$ into Equation 2, for calculation of $\xi$.

An additional complication arises from the fact that, for most synapses, the amplitude, $h$, is not a constant but rather a stochastic variable with a certain distribution. Fortunately, this amplitude distribution of mEPSCs can be measured at the calyx of Held (Borst and Sakmann, 1996; Chuhma and Ohmori, 1998; Schneggenburger et al., 1999). Assuming that the different size classes of mEPSCs occur statistically independently and that no correlation exists between size and release probability, the quantities $h, h^{2}, h^{3}$, and $h^{4}$ in Equations 2, 3, and 6 can be replaced by their expectation values (Fesce, 1990), and we arrive at the following:

$$
\langle h\rangle=\frac{\lambda_{3}^{\prime}}{\left(\lambda_{2}^{\prime}-i^{\prime} I_{p}\right)} \cdot \frac{\left\langle h^{2}\right\rangle\langle h\rangle I_{2}^{\prime}}{\left\langle h^{3}\right\rangle I_{3}^{\prime}} \equiv h_{s}^{\prime} \cdot H_{s}^{\prime}
$$

and

$$
\xi=\frac{\left(\lambda_{2}^{\prime}-i^{\prime} I_{p}\right)^{3}}{\lambda_{3}^{\prime 2}} \cdot \frac{\left\langle h^{3}\right\rangle^{2}}{\left\langle h^{2}\right\rangle^{3}} \cdot \frac{I_{3}^{\prime 2}}{I_{2}^{\prime 3}} \equiv \xi_{s}^{\prime} \cdot Z_{s}^{\prime}
$$

Here, we have introduced the quantities $h_{s}^{\prime}$ and $\xi_{s}^{\prime}$, which, respectively, represent estimates proportional to $h$ and $\xi$ from the skew of the filtered records. Both $h_{s}^{\prime}$ and $\xi_{s}^{\prime}$ have to be multiplied by calibration factors $H_{s}^{\prime}$ and $Z_{s}^{\prime}$, which, according to Equations 10 and 11, are given by the following:

$$
H_{s}^{\prime}=\frac{\left\langle h^{2}\right\rangle\langle h\rangle}{\left\langle h^{3}\right\rangle} \cdot \frac{I_{2}^{\prime}}{I_{3}^{\prime}}
$$

and

$$
Z_{s}^{\prime}=\frac{\left\langle h^{3}\right\rangle^{2}}{\left\langle h^{2}\right\rangle^{3}} \cdot \frac{I_{3}^{\prime 2}}{I_{2}^{\prime 3}} .
$$

Equivalent equations and definitions $\left(H_{4}^{\prime}, Z_{4}^{\prime}\right.$; see legend of Table 1) can be introduced to replace Equations 7-9 for obtaining estimates from the fourth cumulant and the skew:

$$
\begin{gathered}
\langle h\rangle=\left(\lambda_{4}^{\prime} / \lambda_{3}^{\prime}\right) \cdot H_{4}^{\prime} \quad H_{4}^{\prime}=\frac{\left\langle h^{3}\right\rangle\langle h\rangle}{\left\langle h^{4}\right\rangle} \frac{I_{3}^{\prime}}{I_{4}^{\prime}} \\
\xi=\left(\lambda_{3}^{\prime 4} / \lambda_{4}^{\prime 3}\right) \cdot Z_{4}^{\prime} \quad Z_{4}^{\prime}=\frac{\left\langle h^{4}\right\rangle^{3}}{\left\langle h^{3}\right\rangle^{4}} \cdot \frac{I_{4}^{\prime 3}}{I_{3}^{\prime 4}} \\
i^{\prime}=\frac{\lambda_{2}^{\prime}}{I_{p}}\left(1-\frac{\lambda_{3}^{\prime 2}}{\lambda_{2}^{\prime} \lambda_{4}^{\prime}} \frac{H_{s}^{\prime}}{H_{4}^{\prime}}\right)
\end{gathered}
$$

Calibration factors $H_{s}^{\prime}, Z_{s}^{\prime}, H_{4}^{\prime}$, and $Z_{4}^{\prime}$ were calculated from the average time course of the mEPSCs (Fig. $1 B$, Table 1) and from the mEPSC amplitude distribution. The moments of the distribution were as follows: $\langle h\rangle=31.1 \mathrm{pA}($ mean $) ;\left\langle h^{2}\right\rangle=1182 \mathrm{pA}^{2} ;\left\langle h^{3}\right\rangle=5.4 \cdot 10^{-32} \mathrm{~A}^{3}$; and $\left\langle h^{4}\right\rangle=$ $2.91 \cdot 10^{-42} \mathrm{~A}^{4}$. The fact that the calibration factors contain the quantity of interest, $\langle h\rangle$, is of little concern, because they do so in combination with the higher moments in a way that they are nondimensional and invariant, when an amplitude distribution uniformly shrinks or expands. Because we are mainly interested in using Equation 9 to study possible postsynaptic desensitization, and, assuming that desensitization scales all of the mEPSC amplitudes by a constant factor, we expect $H_{s}^{\prime}$ to be constant during our recordings.

In practice, the calibration factors $H_{s}^{\prime}$ and $Z_{s}^{\prime}$ are readily calculated from the equations above, provided that the time course of an individual mEPSC and its amplitude distribution is known (see legends of Fig. 1 and Table 1). Alternatively, they can be determined from simulated data, using the measured amplitude distribution for the simulation (see below) and applying exactly the same routines, as used for the analysis of experimental postsynaptic currents. In two previous papers (Neher and Sakaba, 2001; Sakaba and Neher, 2001), we used this latter method to obtain relative values of mEPSCs amplitudes. In the present work, we provide calculated values throughout, using mainly Equations 10-16.

\section{MATERIALS AND METHODS}

Analysis. Postsynaptic, as well as presynaptic, currents were sampled at rates of $20 \mathrm{kHz}$ after low-pass filtering at either 2.9 or $6 \mathrm{kHz}$ and using a partial hardware series resistance compensation (see below for record- ing conditions). The remaining series resistance (typically 2-3 M $\Omega$ ) was compensated by software using a routine similar to that of Traynelis (1998). In this manner, $100 \%$ compensation was readily obtained. Analysis was performed with custom-written macros in the environment of IGOR-Pro (WaveMetrics Inc., Lake Oswego, OR). The deconvolution method was performed as described by Neher and Sakaba (2001). The Igor macros used are available on our departmental homepage (www.mpibpc.gwdg.de/abteilungen/140/software).

A first type of fluctuation analysis was performed on single traces of postsynaptic currents by calculating sliding averages of the mean, variance, skew, and fourth cumulant (averaged over a specified analysis window). For the latter three quantities, the data were band-pass filtered before calculating the moments. Variance and skew were set equal to the second and third moment. The fourth cumulant was calculated according to Equation 6 from the fourth moment and the variance after smoothing these quantities by the sliding window. This order of processing is important, because Equation 6 involves a nonlinear operation. Likewise, the quantities $h_{s}^{\prime}$ and $\xi_{s}^{\prime}$ (from Eqs. 10 and 11) or the corresponding quantities $h_{4}^{\prime}$ and $\xi_{4}^{\prime}$ (estimates from the fourth cumulant and skew) were calculated after smoothing. For this type of analysis, the sliding windows had to be relatively large and the traces had to be reasonably stationary within the length of a window (see Results for an analysis of the effects of nonstationarity).

For most types of experiments, however, we wanted to analyze records with quite pronounced nonstationarities. Typically, we had 5-10 such traces with very similar mean time courses available. It was then possible to improve the analysis by a method similar to "ensemble noise analysis," introduced by Sigworth (1980) for nonstationary records of voltagedependent currents. This technique analyzes fluctuations around the mean of an ensemble. The most effective way of removing long-term trends is to subtract subsequent records from one another. The variance of such difference records is twice the variance around the ensemble mean (Heinemann and Sigworth, 1993). For the skew, such simple subtraction is not possible, because the expected skew of a difference between two similar records is zero. However, the difference between an individual record and the sample mean over $N$ such records is quite suitable for analysis. Such a difference record is given by the following:

$$
\Delta y_{i}=y_{i}-\frac{1}{N} \sum_{v=1}^{N} y_{v}
$$

and the expectation value for its skew, $\lambda_{3 D, i}$ (given statistical independence among traces), is as follows:

$$
\begin{aligned}
\lambda_{3 D, i} & =\left(1-\frac{1}{N}\right)^{3} \lambda_{3}-(N-1) \frac{1}{(N)^{3}} \lambda_{3} \\
& =\lambda_{3}\left(\frac{N-1}{N}\right)^{3}\left(1-\frac{1}{(N-1)^{2}}\right),
\end{aligned}
$$

where $\lambda_{3}$ is the skew around the true ensemble mean. When $N$ is greater than 5 , the multiplier of $\lambda_{3}$ is larger than 0.5 , and, thus, a substantial fraction of the skew is recovered. The corresponding equations for variance $\left(\lambda_{2 D}\right)$ and fourth cumulant $\left(\lambda_{4 D}\right)$ are as follows:

$$
\lambda_{2 D, i}=\lambda_{2}\left(\frac{N-1}{N}\right)
$$

and

$$
\lambda_{4 D, i}=\lambda_{4}\left(\frac{N-1}{N}\right)^{4}\left\{1+\frac{1}{(N-1)^{3}}\right\} .
$$

The advantage of this type of ensemble analysis is that it tolerates substantial nonstationarities of the original records. To efficiently reduce slow trends between records, we subtracted scaled and shifted versions of the sample mean record. Scalers and shift parameters for individual records were determined by minimizing the mean square deviation between a given record and the ensemble mean over a suitable time window. Thereby, trends and transient artifacts are eliminated but results are not modified in any other way, provided that scalers stay within the range of $0.8-1.2$ (see below). The time window for determining the scalers should be selected from a region of the mean trace in which the largest nonstationarities occur (or else the nonstationary region of interest), and it should be long enough that it contains a large number of 
random events in any one trace. The latter requirement derives from the fact that the least-square optimization procedure reduces the number of degrees of freedom of the fluctuations by two.

The combined effect of high-pass filtering of the current records and of subtracting the weighted mean made the analysis quite independent of nonstationarities, as will be documented by simulations below.

Filtering. Digital filtering was performed using combinations of smoothing routines supplied by IGOR, as follows. The rationale for the particular combinations will be given in Results. For low-pass filtering, a sliding average using the "Box-Smooth" function with a specified averaging window $T_{1}$ (usually $0.3 \mathrm{msec}$ ) was used. To remove the secondary maxima of the resulting sinx/x-type filtering characteristics, the first smoothing was followed by a second smoothing with a window $0.8 \times T_{1}$, resulting in a filter function with a $-3 d D$ point at $f_{o}=0.5 / T_{1}(=1670 \mathrm{~Hz}$ for $T_{1}=0.3 \mathrm{msec}$ ), which dropped to values lower than $-60 \mathrm{db}$ at $\sim 1.6 \cdot f_{o}$. Also, secondary maxima were lower than $-50 \mathrm{db}$. For high-pass filtering, a low-pass-filtered record (see above) was subtracted from unfiltered data. Two passes of such high-pass filtering were performed on all records, one using a window $T_{h}$ and a second one using $8 \cdot T_{h}$. In the first pass, the low-pass-filtered record was shifted by $T_{h} / 2$ before subtraction (to preserve the asymmetry of the waveform), and, in the second pass, it was shifted by $-4 T_{h}$. For band-pass filtering, low-pass filtering and high-pass filtering were applied sequentially. The choice of the smoothing windows $T_{1}$ and $T_{h}$ for low- and high-pass filtering, respectively, will be explained in the context of the simulations described below. The filter characteristics used for most of the variance and skew analyses is shown in Fig. 1. It was calculated with $T_{1}=T_{h}=0.3 \mathrm{msec}$. Note that a different filter procedure was used by Neher and Sakaba (2001) and Sakaba and Neher (2001).

Simulation. The purpose of the simulation was to provide traces for a given set of model assumptions and a specified time course of the release rate $r(t)$. The corresponding IGOR macros were written such that either experimental release time courses [as derived from deconvolution (Neher and Sakaba, 2001)] or appropriate test functions (this paper) could be specified. The macros build the output trace as a sum of miniature currents progressing from beginning to end. For each sample interval, $\Delta t$, the number of release events, $n_{R}$, was determined as the output of a random number generator with a Poisson distribution and the mean number of events equal to $r(t) \cdot \Delta t$. If $n_{R}$ was greater than 0 , an mEPSCtype waveform (as specified by model parameters) was added to the output trace with its origin at the given sample point. The amplitude of that addition was determined as a sum of $n_{R}$ random numbers drawn from a random number generator with the distribution of the mEPSC amplitudes. In effect, this procedure merged the mEPSCs originating from one sample interval into a single one. This simplification is considered to be valid, if the sample interval (typically $50 \mu \mathrm{sec}$ ) is much shorter than the mEPSC rise time (200-300 $\mu \mathrm{sec})$. The simulation also tracked the mean residual glutamate concentration, $C_{D}$, in the synaptic cleft. This was assumed to be a convolution of the released amount equal to the following:

$$
C_{D}(t)=\int_{o}^{t} c_{r}\left(t-t^{\prime}\right) r\left(t^{\prime}\right) d t^{\prime},
$$

where $r\left(t^{\prime}\right)$ is the release rate and $c_{r}(t)$ is a diff usion-type kernel, given by (Crank, 1975):

$$
c_{r}(t)=\frac{1}{4 \pi D t^{n_{D}}} \cdot \exp \left(-x^{2} /(4 \pi D t)\right)
$$

The residual current, $I_{D}$, which was added to the sum of mEPSC currents, was assumed to be as follows:

$$
I_{D}=\beta \cdot C_{T}(t)^{n} .
$$

In a previous publication (Neher and Sakaba, 2001), it has been shown that this formalism allows one to fit glutamatergic postsynaptic currents over a wide range of experimental situations. The choice was explained in that paper; here we just list the parameters and give typical values. The peak amplitude of mEPSC was calculated according to the measured distribution with a mean of $32.1 \mathrm{pA}$. Its time course was assumed to be a double or triple exponential sum with the following: $\tau_{\mathrm{r}}$ of $0.2 \mathrm{msec}$, rise time constant; $\tau_{1}$ of 2 msec, decay time constant; $\alpha$ of 0 or 0.2 , fraction of a second slow component of decay; and $\tau_{2}$ of $10 \mathrm{msec}$ time constant of slow decay phase. These values are typical for recordings in the presence of $100 \mu \mathrm{M}$ cyclothiazide (which was used in most recordings to prevent glutamate receptor desensitization).

The residual current was described by an amplitude factor, selected such that residual current correctly reproduced experimental currents at long times after release. The parameters were as follows: $n=1.2$ exponent of the power law of glutamate channel activation; $r_{D}=0.76 \mu \mathrm{m}$ diffusional distance; $n_{D}=0.9$ exponent of the diff usion law; and $D=30$ $\mu \mathrm{m}^{2} / \mathrm{sec}$, diff usion coefficient of the transmitter.

For the analysis of simulated records, the resulting sum of mEPSCderived and residual currents was filtered and processed in the same way as experimental traces. It should be noted that most of the simulation did not include noise from channel open-close fluctuations and background recording. If required, such "channel noise" was introduced by adding to the simulated trace a real current trace obtained from an experiment in which glutamate-activated current was elicited by bath application of 100 $\mu \mathrm{M}$ S-AMPA.

Simulation of mEPSCs as a superposition of single channel currents. For some of the simulations, we constructed simple mEPSCs with mean time courses rising with $\tau_{0}$ and decaying exponentially with $\tau_{1}$ by activating channels at a rate that has an initial value of $A_{0}$ and decays exponentially with time constant $\tau_{0}$. After initial activation, channels fluctuate between an open state and a short-lived closed state, $C_{s l}$, according to the following scheme:

$$
\longleftarrow_{k_{-1}} C_{s l} \stackrel{\beta}{\stackrel{\beta}{\Leftrightarrow}} O
$$

The rates and $k_{-1}$ were selected such that bursts of openings were obtained with a mean burst duration of $2 \mathrm{msec}$, consisting of flickers with a mean duration of $0.5 \mathrm{msec}$ and mean gaps of $0.2 \mathrm{msec}$. The corresponding parameters are as follows: $k_{-1}=1590 \mathrm{sec}^{-1}, \beta=3409 \mathrm{sec}^{-1}$, and $\alpha=$ $2000 \mathrm{sec}^{-1}$ (Colquhoun and Hawkes, 1981). We chose the amplitude of the single channel current such that the mean current during the burst was $1.5 \mathrm{pA}$, and we chose $A_{\mathrm{o}}$ such that the mean mEPSC amplitude was $32 \mathrm{pA}$. This simple model is not intended to reproduce the complicated and controversial open-close kinetics of AMPA-activated currents (Partin et al., 1996; Rosenmund et al., 1998) but only to estimate the errors made by assuming statistical independence of channel gating fluctuations and release statistics (see Results).

Physiological recordings. Presynaptic and postsynaptic recordings at the calyx of Held were performed in the slice preparation of the rat brainstem as has been described previously (Neher and Sakaba, 2001). Briefly, 8- to 10-d-old Wistar rats were decapitated without anaesthesia according to local guidelines. The brainstem was immersed in ice-cold, lowcalcium saline, which contained (in mM): $125 \mathrm{NaCl}, 2.5 \mathrm{KCl}, 0.1 \mathrm{CaCl}_{2}$, $3 \mathrm{MgCl}_{2}, 25$ glucose, $1.25 \mathrm{NaHCO}_{3}, 0.4$ ascorbic acid, 3 myo-inositol, and 2 Na-pyruvate, $\mathrm{pH}$ 7.3-7.4 (320 mOsm; bubbled with $95 \% \mathrm{O}_{2}$ and 5\% $\left.\mathrm{CO}_{2}\right)$. Transverse slices of the brainstem (150-200 $\mu \mathrm{m}$ thick) were cut using a vibratome. Slices were incubated in the chamber for at least 30 min at $36^{\circ} \mathrm{C}$ in normal extracellular solution while being continuously bubbled with $95 \% \mathrm{O}_{2}$ and $5 \% \mathrm{CO}_{2}$. Normal extracellular solution was the same as the low-Ca ${ }^{2+}$ saline, except that $2.0 \mathrm{~mm} \mathrm{CaCl}$ and $1.0 \mathrm{~mm}$ $\mathrm{MgCl}_{2}$ were used and $100 \mu \mathrm{M} \mathrm{CTZ}$ and/or $1 \mathrm{~mm}$ kynurenic acid (Kyn) was added, as indicated. Experiments were done within $4 \mathrm{hr}$ after preparation of the slices. All recordings were done at room temperature $\left(\sim 21-24^{\circ} \mathrm{C}\right)$. A presynaptic terminal and a postsynaptic target were simultaneously clamped at $-80 \mathrm{mV}$ with patch pipettes. The presynaptic pipette (4-6 M $\Omega$ ) was filled with a solution containing (in $\mathrm{mM}$ ): 125-130 Cs-gluconate, 20 tetraethylammonium (TEA)-Cl, 10 HEPES, $5 \mathrm{Na}_{2}$ phosphocreatine, $4 \mathrm{MgATP}, 0.3 \mathrm{GTP}$, 0.5 EGTA, pH 7.2 with $\mathrm{CsOH}$ (310 mOsm). The postsynaptic pipette $(2-3.5 \mathrm{M} \Omega$ ) was filled with the same solution as the presynaptic pipette, except that the concentration of EGTA was increased to $5 \mathrm{~mm}$.

During recordings, 0.5-1 $\mu \mathrm{M}$ TTX, $10 \mathrm{~mm}$ TEA-Cl, $0.1 \mathrm{~mm} 3,4-$ diaminopyridine, and $50 \mu \mathrm{M}$ D-AP-5 were added to the normal extracellular solution to isolate the presynaptic calcium current and to block NMDA receptors. This way, AMPA receptor-mediated EPSCs were isolated to monitor quantal release. TTX was purchased from Alomone Labs (Jerusalem, Israel). S-AMPA, D-AP-5, and CTZ were from Tocris Cookson (Köln, Germany). Other drugs were from Sigma (Deisenhofen, Germany).

Both presynaptic and postsynaptic cells were whole-cell clamped to a holding potential of $-80 \mathrm{mV}$, using EPC9/2 amplifiers controlled by the Pulse program (Heka Elektronik, Lambrecht, Germany). No liquid junc- 
tion potential correction was applied. Thirty to $70 \%$ of the presynaptic series resistance $\left(R_{s}\right.$ of 8-30 M $\Omega$, typically $15 \mathrm{M} \Omega$ ) was compensated. The postsynaptic series resistance (3-8 M 2 , typically $5 \mathrm{M} \Omega$ ) was compensated so that the uncompensated series resistance was $\sim 2-3 \mathrm{M} \Omega$. This remaining series resistance was software-compensated off-line, as described above.

\section{RESULTS}

\section{Simulations}

Optimizing the filtering procedure for high signal-to-noise ratio and tolerance against nonstationaries

Optimal filtering of postsynaptic currents depends on a number of criteria, such as suppression of background noise (including channel noise), optimization of the ratio between the mean of the estimated cumulants and their intrinsic statistical variation, suppression of trends and nonstationarities in the data, and preservation of asymmetry of the original signal (for skew). The dependence of noise estimates on bandwidth can be derived analytically, and, for many reasons, high-pass filtering with a high cutoff frequency is beneficial (Fesce, 1990). However, at very high frequencies, the noise power originating from the mEPSC time course decreases rapidly, whereas background noise, such as amplifier noise or ion channel noise, increases or stays constant. Therefore, an optimal frequency band for filtering has to be found. In the following, we will first discuss some theoretical expectations and then search for optimal parameters by simulation.

We are interested in determining the relative accuracy of our estimates of the cumulants and want to optimize the filtering procedure so that the coefficient of variation $(\mathrm{CV}), \sigma_{\lambda_{v}^{\prime}} / \lambda_{v}^{\prime}$, is as small as possible for a given observation interval. Neher and Sakaba (2001) pointed out that the coefficient of variation of the variance is independent of the frequency of elementary events and is proportional to $\sqrt{2 / n}$, where $n$ is the number of independent samples that can be obtained during the observation interval $T$. This, in turn, depends on the filtering, given the requirement that the observation for obtaining such a sample has to be longer than the duration of the filtered elementary event. For this reason, the filtering should be done such that the filtered elementary event has the shortest possible half-width. This requirement for short half-width is even more serious for the skew, as pointed out by Segal et al. (1985). The argument calls for high-pass filtering (which eliminates the residual current and reduces the slow decay phase of mEPSCs) with a corner frequency as high as possible. Additionally, high-pass filtering eliminates trends and other nonstationarities in the record. However, when examining noise power spectra of stationary records derived from either a stream of mEPSCs or currents evoked by superfusion of AMPA, it is noticed that channel and instrumentation noise may very well dominate noise spectral density at frequencies above $1 \mathrm{kHz}$. Also, the spectral density of the mEPSC-derived signal at such high frequencies critically depends on the mEPSC rise time, which, in turn, may be dependent on clamp speed and series resistance of the postsynaptic recording. Therefore, we restricted the band pass on the high end to $\sim 1.6 \mathrm{kHz}$, which corresponds to a smoothing window $T_{1}$ of $0.3 \mathrm{msec}$ (see Materials and Methods), or 1.5-2 times the estimated mEPSC rise time (see paragraph on filtering, above). With this restriction, a relatively narrow bandpass (implemented by software, as shown in Fig. 1A), appeared to be optimal. We also restricted the filter kernel to as few points as possible to avoid contamination of those parts of the record, which are relatively stationary, by large transients of current in neighboring segments. This, too, is a property of the software
A

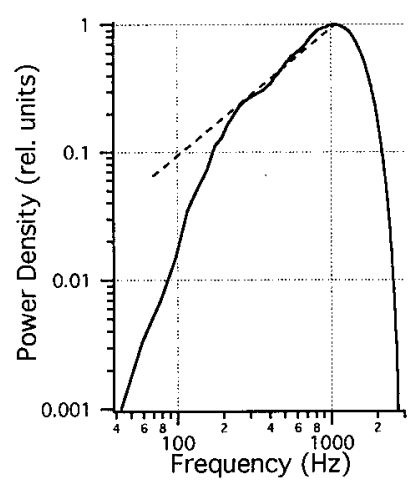

B

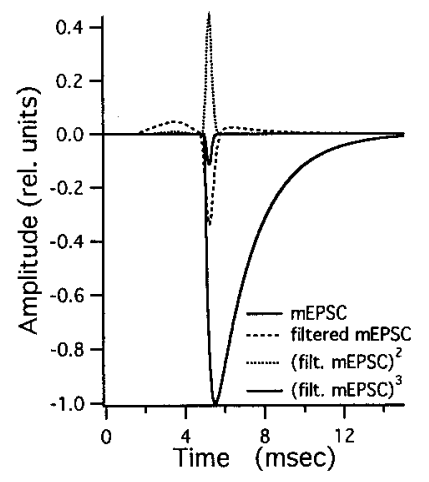

Figure 1. The bandpass filter. $A$, Filter characteristics used for most of the bandpass filtering in the fluctuation analysis. Five thousand points of Gaussian white noise (from Igor function gnoise) were interpreted as a current record with $50 \mu \mathrm{sec}$ sample interval and bandpass filtered with a low-pass window $T_{1}$ of $0.3 \mathrm{msec}$ and a high-pass window $T_{h}$ of the same length. Spectral analysis was performed using the Igor Routine PSD with a segment length of 1024 points and a Hanning-type window. The filter characteristic has its maximum at $1074 \mathrm{~Hz}$. The $-3 \mathrm{db}$ point is at $1670 \mathrm{~Hz}$. The broken line represents the curve f/1074 and indicates that the filter curve is well fitted by a single pole high pass $(-3 \mathrm{db}$ point at $537 \mathrm{~Hz})$ between $200 \mathrm{~Hz}$ and $1 \mathrm{kHz}$. B, Time course of a typical mEPSC, with a 0.2 msec time constant and a 2 msec decay time constant. For simulations, this standard mini was multiplied by an amplitude, as drawn from the amplitude distribution. Also included in this figure are the filtered mEPSC and the filtered mEPSC raised to the second and third power. The latter two curves are expanded five times for better visibility. By numerical integration, the following values are obtained from Equations $1-3: I_{2}^{\prime}=4.3 \cdot 10^{-5}, I_{3}^{\prime}=1.06 \cdot 10^{-5}$, and $I_{4}^{\prime}=3.156 \cdot 10^{-6} \mathrm{sec}$.

filter described in Materials and Methods, as shown in Fig. $1 B$. The filter converts an mEPSC into a pulse with $\sim 0.5 \mathrm{msec}$ half-width, preceded and followed by shallow depressions that extend over $\sim 5 \mathrm{msec}$. Fig. $1 B$ also shows that the square and the cube of the filtered signal are narrow pulses with hardly any components preceding and following. In the following simulations, we explore the properties of fluctuation analysis of records filtered in such a way.

\section{Averaging over 500 msec of a rather wavy record gives useful} information on the variance, skew, and fourth cumulant

Postsynaptic current traces were simulated as described in Materials and Methods section. For the first series of simulations, we used the mEPSC parameter set (described in Materials and Methods) without a second decay component ( $\alpha$ of 0 ) and without a residual current ( $\beta$ of 0 ). These parameters are appropriate for recordings in the presence of $100 \mu \mathrm{M} \mathrm{CTZ}$.

First, we simulated stretches (500 msec each) of superimposed mEPSCs at constant release rates of $0.5,1,2,8,12$, and 24 events/msec. Example excerpts from such simulated traces before and after filtering are shown in Figure 2, $A$ and $B$. We calculated the variance, skew, and fourth moment of the filtered records (mean of 0) and formed averages over individual records (500 msec each). We repeated this procedure 50 times and obtained mean values and SDs (given in Table 1, top seven rows). Also given in Table 1 are the estimates for mEPSC amplitude and release rates as derived from the skew and variance (according to Eqs. 10 and 11) using quantities $H_{s}^{\prime}$ and $Z_{s}^{\prime}$ (given by Eqs. 12 and 13). The same estimates based on the fourth cumulant and skew are also shown (Eqs. 14 and 15). Estimates of the mean mEPSC amplitudes (32.1 pA), as well as the release rates, are reproduced 
A

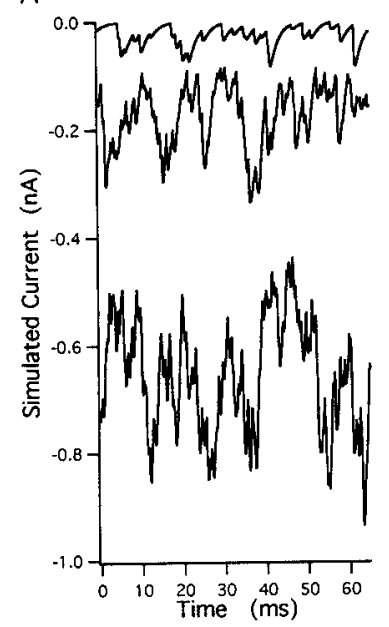

B

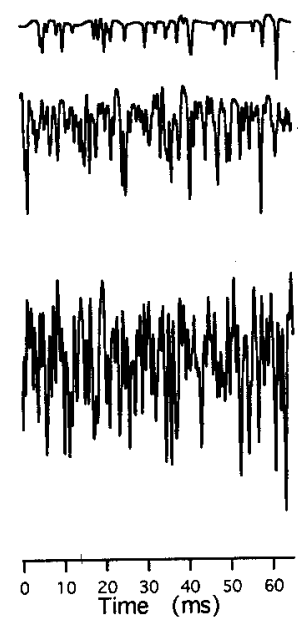

Figure 2. Simulated current traces. $A$, Excerpts from simulated traces at rates of 0.5 (top), 2 (middle), and 8 (bottom) events/msec. mEPSCs had rise time constants of $0.2 \mathrm{msec}$, decay time constants of $2 \mathrm{msec}$, and a peak amplitude distribution. $B$, Same segments of traces as in $A$ after bandpass filtering. Traces are offset to match the DC level of the corresponding traces in $A$.

quite accurately by the analysis based on skew and variance. In contrast, estimates deviate for rates above 2 events/msec when the analysis is instead based on the fourth cumulant (Table 1, Fig. $3 A$ ). This is because at higher frequency, according to the central limit theorem, the distribution of current values approaches a Gaussian, which has a relatively small fourth cumulant.

Coefficients of variation, calculated from the means and SDs of Table 1, are plotted in Figure 3, $B$ and $C$. As expected from the consideration above, the estimates for the coefficient of variation of the variance are almost independent of the mEPSC frequency for rates greater than 2 events/msec. The same is also observed for estimates of the fourth moment. However, the accuracy of the fourth cumulant and of estimates derived from it deteriorate at rates greater than 2 events/msec. The skew is significantly less well resolved than the variance, and its resolution decreases for rates greater than 5 events/msec (Segal et al., 1985). Both the variance and (even more so) the fourth moment are less well resolved at very small rates, probably because their distributions are far from Gaussian, even if averaged over long time intervals. The coefficients of variation of rates and amplitudes reflect the properties of the moments from which they are derived (Fig. $3 C$ ) (see also the theoretical predictions; Fesce, 1990). The rate estimate derived from the fourth moment is without any significance for rates greater than 2 events/msec. Amplitudes and rates estimated from the skew and variance are optimally resolved at rates between 1 and 2 events/msec and are quite accurate over the entire frequency range studied.

Performing the same analysis on segments of different lengths or averaging values from several similar traces readily demonstrates that the coefficient of variation changes with $1 / \sqrt{T}$ (Neher and Sakaba, 2001), where $T$ is the total recording time over which the cumulants are averaged. Thus, for each estimate, the total recording time necessary for a desired accuracy can be calculated. This recording time is $70 \mathrm{msec}$ for variance when $20 \%$ accuracy is desired (with release rates of 2 events $/ \mathrm{msec}$ ) and much longer for the other estimates. For example, an estimation of the mEPSC amplitude with $20 \%$ accuracy requires only $110 \mathrm{msec}$, signifi-

cantly less than a similar estimate for the release rate $(280 \mathrm{msec})$. This difference is expected, realizing that higher powers of cumulants enter the equation for release rate (Eq. 11) than that for amplitude (Eq. 10).

Second, we wanted to know how sensitive such estimates would be to slow variations in the release rate. To this end, we simulated traces with a mean rate of 2 events/msec that increased and decreased sinusoidally by $\pm 50 \%$ with a period of $50 \mathrm{msec}$. The result for the mean cumulants are shown (Table 1) for comparison with the values for a constant release process. Within the statistical error it is seen that the values agree with those corresponding to a constant rate of 2 events/msec. Thus, we confirm that bandpass filtering effectively eliminates the effects of nonstationarities (Fesce, 1990), if the nonstationarities are sufficiently slower than the low frequency cutoff of the filter. Below, we will present in more detail the effects of more rapid transitions in release rates.

Third, we simulated traces with a constant rate of 2 events $/ \mathrm{msec}$ and a fourfold-reduced cutoff frequency of the high-pass branch of the filter. Variance and higher cumulants were increased sevenfold to 30 -fold (Table 1, compare row two, row nine), whereas the estimates of rate and amplitude were still correct, although significantly more noisy. This analysis shows that high-pass filtering should be done with the highest corner frequency that is compatible with other constraints.

\section{Contributions by slow decay components, residual current, and channel noise}

So far, the simulations were performed with a single component mEPSC, in which fluctuations attributable to opening and closing of channels were not considered. We now proceed to simulations in which we include a slow component of mEPSC decay, as well as channel fluctuations. We demonstrate that these additions lead only to small changes in the mean values, provided that their contributions are slow relative to the bandpass of the filter.

When a slow decay component of $20 \%$ amplitude and $10 \mathrm{msec}$ time constant was added in simulations at a release rate of two per millisecond, the mean current at steady state was about twice as large as that for a single component mEPSC. If we included a residual current component, using parameters typical for the calyx of Held (see Materials and Methods) (Neher and Sakaba, 2001), the steady-state currents were increased approximately fivefold. Nevertheless, the variance was unchanged and the skew increased only insignificantly (see Table 1 for simulations at 0.5 , 2,8 , and 24 events/msec). Correspondingly, amplitudes and rates estimated from the skew and variance were correct. A similar result was obtained when we added a slow negative component, producing an undershoot.

We did two kinds of simulations to examine the influence of channel noise. In the first simulation, we simply added current traces recorded during S-AMPA bath application (at $100 \mu \mathrm{M}$ ) from a postsynaptic neuron to the simulated traces at $0.5,2,8$, and 24 events/msec. To do so, we selected experimental traces that had the same mean current as the simulated ones. As expected, the variance of the summed records was increased (Table 1), and the skew and fourth cumulant were changed only insignificantly. More importantly, the SD of all estimates changed very little. The contribution of channel noise to most of the traces was $\sim 15 \%$ of the total variance. When the variance of the AMPA-induced current was measured directly (after bandpass filtering) and plotted against mean current, a straight line was usually obtained. However, the slopes of such lines (corresponding to the parame- 


\begin{tabular}{|c|c|c|c|c|c|c|c|c|c|}
\hline Condition & $\begin{array}{l}\text { Rate } \\
\text { (events/ } \\
\text { msec) }\end{array}$ & $\begin{array}{l}\text { Variance } \\
\left(\mathrm{pA}^{2}\right)\end{array}$ & $\begin{array}{l}\text { Skew } \\
\left(-10^{-34} *\right. \\
\left.\mathrm{A}^{3}\right)\end{array}$ & $\begin{array}{l}\text { 4th Moment } \\
\left(\mathrm{A}^{4} *\right. \\
\left.-10^{-45}\right)\end{array}$ & $\begin{array}{l}\text { 4th Cumu- } \\
\text { lant }\left(\mathrm{A}^{4} *\right. \\
\left.-10^{-45}\right)\end{array}$ & $\begin{array}{l}\text { AmpS } \\
(-p A)\end{array}$ & $\begin{array}{l}\text { Amp 4th } \\
(-p A)\end{array}$ & $\begin{array}{l}\text { RateS } \\
\text { (events/msec) }\end{array}$ & $\begin{array}{l}\text { Rate 4th } \\
\text { (events/msec) }\end{array}$ \\
\hline One-component mEPSC & 0.5 & $26 \pm 2$ & $3.0 \pm 0.5$ & $7 \pm 2$ & $5 \pm 2$ & $32.1 \pm 3.4$ & $32.0 \pm 6.4$ & $0.5 \pm 0.1$ & $0.5 \pm 0.2$ \\
\hline One-component mEPSC & 1 & $50 \pm 4$ & $5.6 \pm 0.9$ & $16 \pm 4$ & $9 \pm 3$ & $30.8 \pm 2.8$ & $30.2 \pm 5.3$ & $1.0 \pm 0.2$ & $1.2 \pm 0.6$ \\
\hline One-component mEPSC & 2 & $103 \pm 8$ & $11.7 \pm 1.9$ & $49 \pm 11$ & $17 \pm 6$ & $31.1 \pm 3.0$ & $27.9 \pm 6.3$ & $2.1 \pm 0.3$ & $3.6 \pm 2.5$ \\
\hline One-component mEPSC & 5 & $255 \pm 11$ & $28.2 \pm 4.1$ & $240 \pm 31$ & $44 \pm 24$ & $30.6 \pm 3.9$ & $29.2 \pm 12.7$ & $5.4 \pm 1.3$ & $24 \pm 60$ \\
\hline One-component mEPSC & 8 & $404 \pm 21$ & $44 \pm 7$ & $553 \pm 68$ & $63 \pm 34$ & $30.3 \pm 4.0$ & $26.7 \pm 12.3$ & $8.7 \pm 2.3$ & $38 \pm 90$ \\
\hline One-component mEPSC & 12 & $606 \pm 31$ & $69 \pm 15$ & $1212 \pm 182$ & $108 \pm 108$ & $31.5 \pm 5.9$ & $27.2 \pm 23.1$ & $12.7 \pm 4.2$ & \\
\hline One-component mEPSC & 24 & $1227 \pm 65$ & $150 \pm 36$ & $4784 \pm 658$ & $258 \pm 269$ & $33.6 \pm 7.0$ & $29.1 \pm 26.6$ & $23.2 \pm 9.2$ & \\
\hline Sinusoidal modulation & 2 & $101 \pm 6$ & $11.1 \pm 1.7$ & $50 \pm 9$ & $19 \pm 6$ & $30.5 \pm 3.4$ & $32.9 \pm 6.7$ & $2.1 \pm 0.4$ & $2.0 \pm 1.2$ \\
\hline Wider bandpass & 2 & $772 \pm 67$ & $172 \pm 46$ & $2388 \pm 674$ & $588 \pm 420$ & $31.1 \pm 6.0$ & $28.0 \pm 13$ & $2.2 \pm 0.7$ & $43 \pm 240$ \\
\hline Two-component mEPSC & 2 & $97 \pm 5$ & $10.31 \pm 0.4$ & $44 \pm 7$ & $15 \pm 5$ & $30.9 \pm 2.9$ & $29.2 \pm 7.1$ & $2.1 \pm 0.3$ & $3.2 \pm 2.2$ \\
\hline Include residual current & 0.5 & $25 \pm 2$ & $2.8 \pm 0.5$ & $6 \pm 2$ & $4 \pm 1$ & $30.6 \pm 3.2$ & $29.5 \pm 5.8$ & $0.5 \pm 0.1$ & $0.6 \pm 0.3$ \\
\hline Include residual current & 2 & $103 \pm 8$ & $12.2 \pm 2.3$ & $53 \pm 13$ & $21 \pm 9$ & $32.6 \pm 4.0$ & $32.9 \pm 8.1$ & $1.9 \pm 0.3$ & $2.29 \pm 1.1$ \\
\hline Include residual current & 8 & $407 \pm 25$ & $45.4 \pm 8.8$ & $573 \pm 90$ & $74 \pm 45$ & $30.6 \pm 4.6$ & $29.9 \pm 14.1$ & $8.8 \pm 2.5$ & \\
\hline Include residual current & 24 & $1227 \pm 69$ & $140 \pm 31$ & $4674 \pm 580$ & $147 \pm 216$ & $31.4 \pm 6.2$ & $17.8 \pm 27.8$ & $26.9 \pm 12.6$ & \\
\hline Plus channel noise & 0.5 & $33 \pm 2$ & $2.8 \pm 0.5$ & $8 \pm 2$ & $4 \pm 1$ & $30.9 \pm 3.4^{a}$ & $28.8 \pm 5.4$ & $0.5 \pm 0.1$ & $0.79 \pm 0.3$ \\
\hline Plus channel noise & 2 & $118 \pm 9$ & $12.1 \pm 2.2$ & $63 \pm 13$ & $21 \pm 9$ & $32.4 \pm 4.1$ & $32.3 \pm 7.9$ & $1.92 \pm 0.4$ & $2.39 \pm 1.2$ \\
\hline Plus channel noise & 8 & $459 \pm 25$ & $44.3 \pm 9.3$ & $703 \pm 97$ & $69 \pm 51$ & $31.5 \pm 5.3$ & $29 \pm 19$ & $7.9 \pm 2.4$ & \\
\hline Plus channel noise & 24 & $1421 \pm 71$ & $135 \pm 33$ & $6191 \pm 665$ & $120 \pm 265$ & $29.6 \pm 6.7$ & $13 \pm 35$ & $32 \pm 19$ & \\
\hline
\end{tabular}

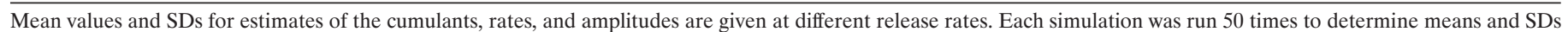

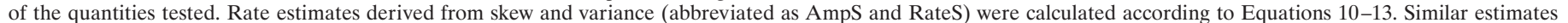

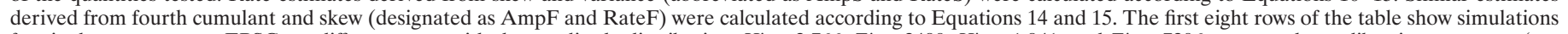

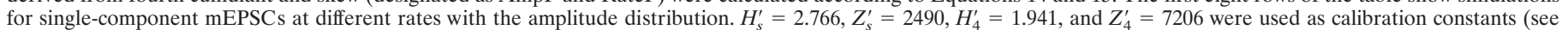

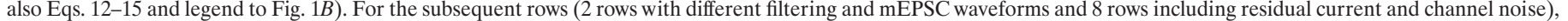

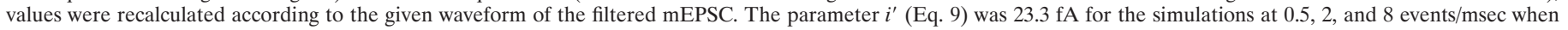

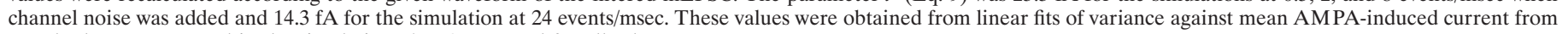
exactly those traces used in the simulation. $i^{\prime}=0$ was used for all other traces.

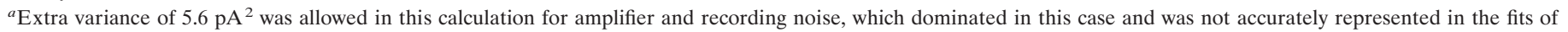
variance against mean of AMPA-induced currents.

ter $i^{\prime}$ in Eqs. 2-10) varied between 12 and $24 \mathrm{fA}$ for different cells (Neher and Sakaba, 2001). When AMPA-induced currents were added to simulated ones, the variance of the superposition was very close to the sum of the individual variances of the AMPA currents and the EPSC simulations. Thus, accurate estimates for amplitudes and rates were obtained when channel variance was accounted for by Equations 10 and 11 (Table 1, last four rows).

In the second simulation, we examined the error that might arise by the assumption of statistical independence between channel noise and the noise originating from the random superposition of mEPSCs. Specifically, we simulated simple mEPSCs explicitly as superpositions of channels (see Materials and Methods), superimposed such mEPSCs randomly at a mean rate of 2 events/msec, and compared the resulting total noise with that of a random superposition of mEPSCs with a fixed time course. Time courses of the latter mEPSCs were similar to that of the mean of the simulated ones. For this simulation, we used a constant mEPSC amplitude, unlike all other simulations. If our assumption about the independence of channel variance, $V_{c}$, and mEPSCs variance (Eq. 2) were correct, we would expect the difference in variance between the two cases to be given by $V_{c}$, according to Equation 2. This is exactly what we found. Variance values in the two simulations differed only by $\sim 20 \%$. This difference $\left(1.9 \cdot 10^{-23} \mathrm{~A}^{2}\right)$ was very close to the variance of another simulation $\left(1.7 \cdot 10^{-23} \mathrm{~A}^{2}\right)$, in which the same type of channels was superimposed randomly at a rate that resulted in the same mean current (176 pA). In the control simulation, the total variance $\left(9 \cdot 10^{-23} \mathrm{~A}^{2}\right)$ was, however, smaller than that of the standard simulation at the same mEPSC rate (Table 1, row three) because, in the latter case, variance is increased by the dispersion of mEPSC amplitudes.

\section{Influence of the dispersion of mEPSC amplitudes}

The heterogeneity of mEPSC amplitudes has a pronounced influence on the estimates for size and rate. The effect on mean values are taken care of by the correction factors described in Equations 10-13. To investigate whether this dispersion influences the coefficients of variation of the estimates and to test the overall correctness of the analysis (with regard to the expectation values), we compared simulations based on the experimental amplitude distribution (described above) with two additional simulations in which the mean amplitudes were close to the mean of the experimental distribution. In one case, the amplitude was fixed at this value (31.1 pA), and, in the other, it had a very large dispersion, with half of the mEPSCs having an amplitude of 12.5 $\mathrm{pA}$ and the other half $52.5 \mathrm{pA}$. In both cases, amplitudes and rates were estimated accurately and with the same coefficients of variation as in control cases (analyses performed at 2 events/msec).

\section{Time resolution of noise estimates}

So far, we have shown that high-pass filtering of the records renders noise estimates relatively insensitive to slow variations in release rate. When we analyze experiments in which the release process changes slowly (such as during slowly decaying, asynchronous release after a strong stimulus), the question arises what the "time resolution" of the estimates will be. We already showed 
A

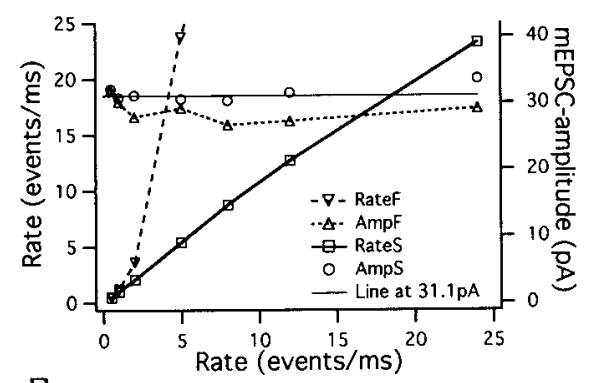

B

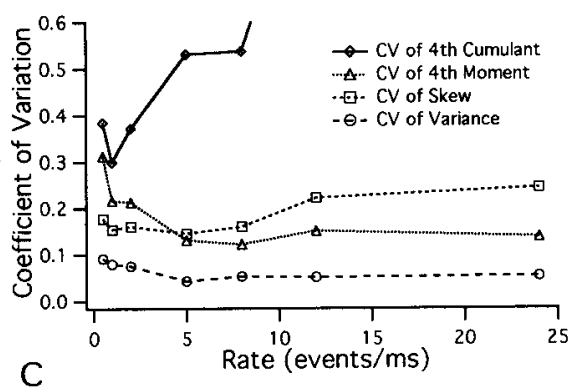

C

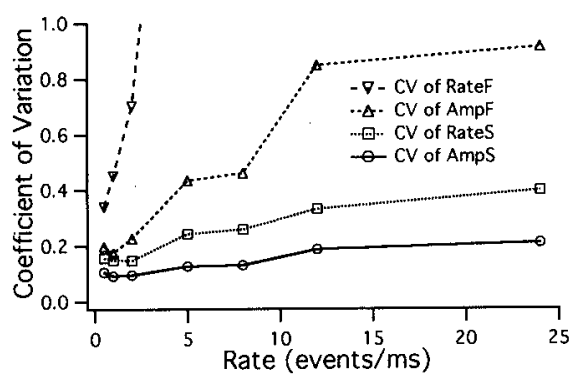

Figure 3. Estimates for rates, mEPSC amplitudes, and the coefficients of variation. Simulated records $500 \mathrm{msec}$ in length were analyzed as described in Materials and Methods. Estimates for the rates and mEPSC amplitudes $(A)$ and coefficients of variation (SD divided by the absolute magnitude of the mean) of cumulants $(B)$ and mEPSC amplitudes and rates $(C)$ are plotted against the rate of occurrence of mEPSCs. Values are taken from Table 1. For abbreviations used in the labeling, see the legend to Table 1.

above that a total recording time of $70 \mathrm{msec}$ is required to estimate the variance with an accuracy of $20 \%$. Likewise, we can ask what recording time is required to achieve an SD of the variance estimate that is equal to its mean. It is reasonable to define this time as the time resolution of the variance estimate, because this duration is the shortest time interval, $T$, for which the estimate bears any meaning. Because the SD of the noise estimates at high enough mEPSC rates varies with $1 / \sqrt{T}$ (when the estimates have a Gaussian distribution), we can calculate the time resolution, $T_{r}$, from values found in Table 1 from the following equation:

$$
T_{r}=T_{0} \cdot C V_{0}^{2}
$$

where $C V_{0}$ is the coefficient of variation ( $\left.\mathrm{SD} / \mathrm{mean}\right)$, and $T_{0}$ is the time window over which the values in Table 1 were obtained (500 msec). Table 2 gives some of the calculated values for the following quantities: variance, AmpS (amplitude, as estimated from skew and variance), RateS (rate from skew, and variance), and the corresponding values for the estimates based on the fourth cumulant and skew (AmpF and RateF). We see that the resolu-
Table 2. Time resolution of noise estimates

\begin{tabular}{llcrrr}
$\begin{array}{l}\text { Rate (events/ } \\
\text { msec) }\end{array}$ & Variance & AmpS & AmpF & RateS & RateF \\
\hline 0.5 & 2.6 & 7 & 17 & 20 & $130^{a}$ \\
2.0 & 2.6 & 8 & $50^{a}$ & $40^{a}$ & $\sim 300^{a}$ \\
8.0 & 1.5 & 15 & $\sim 400^{a}$ & $110^{a}$ & \\
24.0 & 1.3 & $50^{a}$ & $>500$ & $\sim 350^{a}$ &
\end{tabular}

Values (in milliseconds) were calculated according to Equation 24 from the four bottom rows of Table 1 and represent simulations under "realistic" conditions, i.e., with residual current and channel noise superimposed. All values were either confirmed by new simulations using the specified time window or else replaced by new numbers (marked as footnote $a$ ) if this test failed.

${ }^{a}$ These values were corrected by a second round of simulations (see Materials and Methods).

tion for variance is as short as $1-3 \mathrm{msec}$, depending on the mEPSC rate. This demonstrates that an order of magnitude estimate for variance can be obtained within surprising short times. The time resolutions for amplitude and rate estimates are within 10 and $100 \mathrm{msec}$, with better values at lower mEPSC rates. It should be noted that the values given in Table 2 are the total recording times necessary. Thus, if there is an ensemble of $n$ similar traces available, the time resolution for the average estimate will be approximately $T_{r} / n$ or else the coefficient of variation for the time interval $T_{r}$ will be $1 / \sqrt{n}$. We performed simulations at 0.5 events/msec over time windows equal to the $T$ values predicted from Equation 24. In approximately half of the cases shown in Table 2, these calculations confirmed Equation 24. However, for estimates involving the fourth cumulant, particularly at large release rates, $T_{r}$ values turned out to be up to two times larger than those extrapolated from the numbers obtained with a 500 msec analysis window (Eq. 24 and Table 1). The reason for the discrepancy is most likely a violation of the assumption (underlying Eq. 24) that the estimates have a Gaussian distribution.

\section{Tolerance towards transient changes in release rate}

The above consideration addressed only the question of how long a recording interval must be to yield meaningful estimates. In addition, we wanted to find out how the estimates are influenced by trends and transient changes in release rates. To this end, we simulated postsynaptic current traces with abruptly changing release rates, subjected these to the same analysis as used above, and tested how well the resulting rate estimates reproduce such transient changes. We averaged estimates over time windows of 5 msec, which is expected to result in a coefficient of variation of $\sim 0.6$ for the variance trace. The parameters for the mEPSCs were selected to resemble those found in recordings from the calyx of Held, and a residual current was included. In agreement with a previous study, in which variance was analysed only (Neher and Sakaba, 2001), we found that variance and skew are capable of tracking changes in release rate, provided that those are not excessive. In fact, the resolution (both in time and amplitude) is quite remarkable, especially if a few similar traces of a given protocol are available for averaging. Single traces provide order of magnitude estimates for variance and release rates with a time resolution of 5-10 msec. Correspondingly, for averages over 10 similar traces time resolution approaches $1.5-3 \mathrm{msec}$. As expected from Figure 3, the coefficient of variation of the skew was larger than that of variance, and that of the amplitude estimate (as derived from skew and variance) was in between. The estimate of the release rate, on the other hand, was not as well 

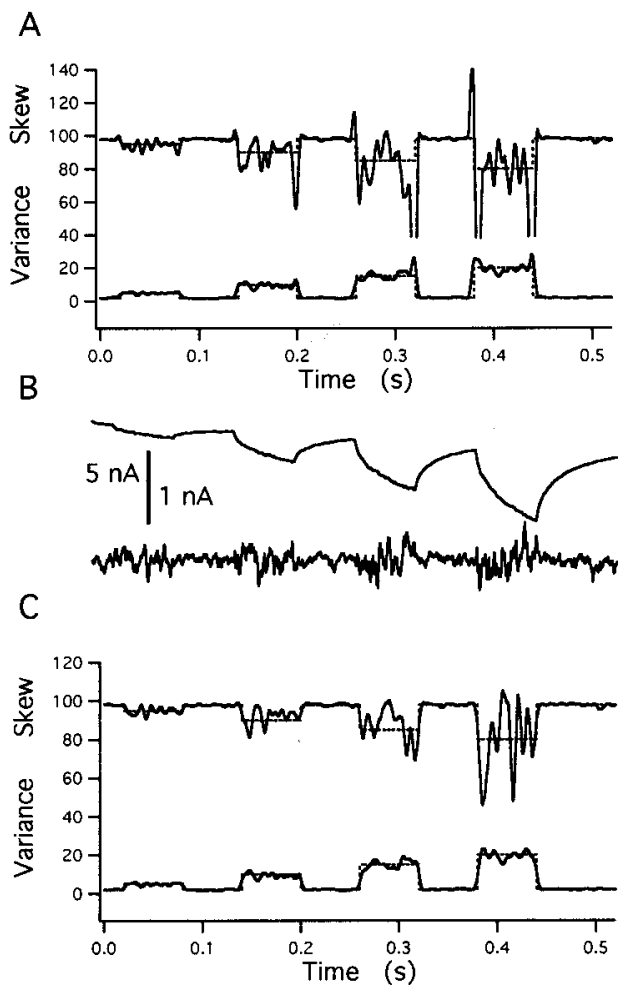

Figure 4. Mean subtraction reduces transient artifacts. Simulation results for four cycles of a square wave release function with increasing amplitudes, averaged over 10 records, are shown. Release rate changes abruptly between a basal rate of 2 events/msec and values of $5,10,15$, and 20 events/msec for $60 \mathrm{msec}$ intervals each. The time course of the release function is shown by the broken lines in $A$ and $C$. $A$, Estimates of the variance and skew scaled such that the absolute magnitude of these quantities is expected to be equal to release rate. Transient artifacts of the top skew trace at $0.32,0.38$, and $0.44 \mathrm{sec}$ are truncated. $B$, Average current of the traces and one example of a mean subtracted trace at higher gain. $C$, The same analysis as $A$, after subtraction of the mean time course from the individual traces (according to Eqs. 18 and 19).

resolved (as expected from Fig. 3 and on the basis of theory; Fesce, 1990). Thus, the best strategy might be to analyze variance and skew together only for mEPSC amplitude. In case that mEPSC amplitude is found to be constant or only slowly varying with time, the rate can be inferred from variance alone by inverting Equation 2 and inserting the known $h$ :

$$
\xi=\left(\lambda_{2}^{\prime}-i^{\prime} I_{p}\right) /\left(h^{2} I_{2}^{\prime}\right)
$$

Averaged simulation results were quite insensitive to variations between individual traces. Thus, results did not change when release rates used for simulations fluctuated by up to $20 \%$ between traces. Transients in the variance or skew during abrupt changes in release rate were greatly reduced, when mean subtraction was applied in an ensemble of records (described in Materials and Methods). Figure 4 compares simulation results with and without mean subtraction. A series of release episodes (each with a square pulse-like release rate at increasing amplitudes between 5 and 20 events/msec) was simulated, and averages over 10 records are displayed: first without (Fig. $4 A$ ) and then after mean subtraction (Fig. $4 C$ ). The mean trace (average of 10 records), as well as an example of a difference trace, is shown in Figure $4 B$. The skew is particularly sensitive to transients. In Figure $4 A$, it is seen that its estimate is out of range for two of the

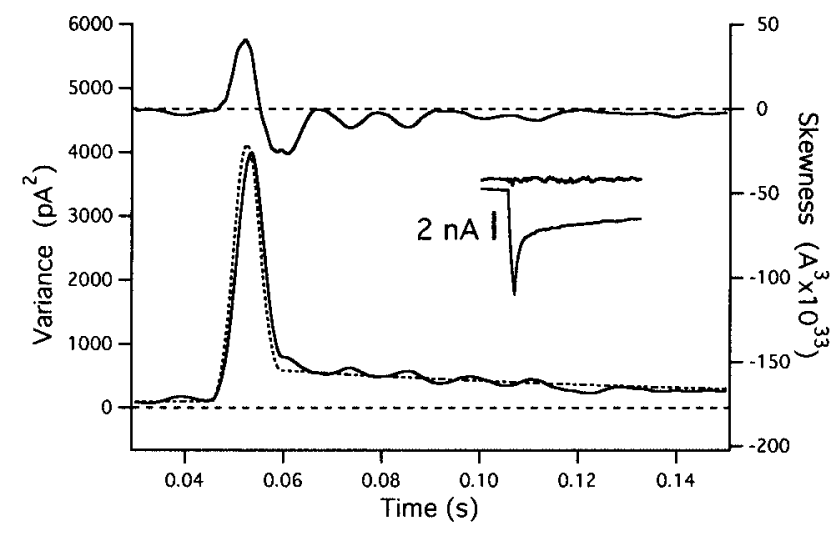

Figure 5. Mean subtraction and trace-to-trace fluctuations. The estimate for variance (bottom curve) is remarkably insensitive to small fluctuations between traces, whereas skewness (top trace) is distorted. The rate function was multiplied by a scaler that varied randomly between 0.8 and 1.2, before starting the simulation of individual traces. The mean of 10 traces was calculated, and an ensemble noise analysis was performed as described in Materials and Methods (Eqs. 18 and 19), with the scaler for the mean subtraction optimized for each individual record. The figure shows the time window (between 30 and $150 \mathrm{msec}$ ) during which the mEPSC rate rises abruptly to a value of 100 events/msec for a period of $5 \mathrm{msec}$. Estimates for variance and skew, as well as the mean rate function, were averaged over a sliding window $5 \mathrm{msec}$ in length. The stippled curve is the smoothed rate function, scaled such that rate and variance are expected to agree. The inset shows the average current trace and an example for an individual difference trace (same time window as other traces).

larger transitions, which is not the case after mean subtraction (Fig. 4C). Variance estimates, on the other hand, were more tolerant to transient changes. An example is given in Figure 5, in which the variance estimate for an abrupt transition from 2 to 100 events/msec and back again is shown. It is free of artifacts, although the rates of individual traces were allowed to fluctuate by up to $20 \%$.

\section{Experiments on the calyx of Held}

Estimation of mEPSCs amplitudes using higher moments

We performed experiments on the calyx of Held synapse to determine the range of stimulation conditions over which the amplitude estimate for mEPSCs, calculated as described above, remains constant and agrees with the directly measured mEPSC. From the results of Neher and Sakaba (2001), it is expected that this is the case under standard conditions only for quite mild, short-lasting stimulation. Any stimulus releasing a substantial fraction of the releasable vesicle pool, however, is expected to cause desensitization and a decrease in the mEPSC size. Although Neher and Sakaba (2001) showed that desensitization is largely blocked by CTZ, their analysis of mEPSC amplitudes was not very reliable when release rates were small $\left(<10 \mathrm{msec}^{-1}\right)$ and stationary in the presence of a large residual current. Therefore, the possibility remains that mEPSC amplitudes were reduced under CTZ during extended stimulation, when release rates dropped toward very low levels and depletion of the release-ready pool of vesicles occurred (Meyer et al., 2001). Then, the size of the release-ready pool of vesicles might in reality be unlimited, which, of course, would imply the breakdown of the vesicle pool concept at the calyx of Held (Schneggenburger et al., 1999; Wu and Borst, 1999; Sakaba and Neher, 2001).

To address these issues, we designed two stimulation protocols. In a first set of experiments the mEPSC amplitude was estimated under mild stimulation conditions, to learn about the applicability 


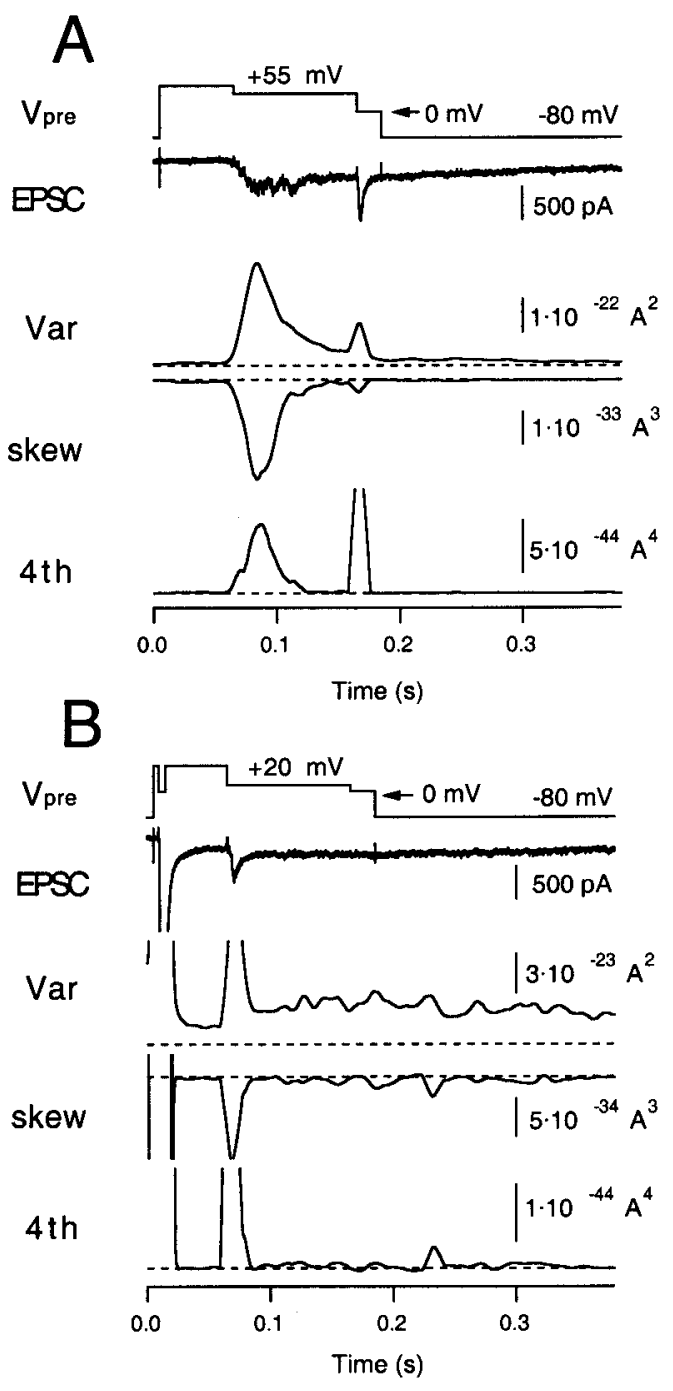

Figure 6. Higher moments measured from an evoked EPSC at the calyx of Held synapse. $A$, The moderate release protocol. The presynaptic terminal was depolarized from -80 to $+80 \mathrm{mV}$ ( $\left.V_{\text {pre }}, 50 \mathrm{msec}\right)$ and then held at $+55 \mathrm{mV}$ for $100 \mathrm{msec}$ to evoke a slowly rising EPSC (moderate release; second trace). The terminal was finally held at $0 \mathrm{mV}$ for $20 \mathrm{msec}$ to evoke maximum $I_{\mathrm{Ca}}$ and to deplete the release-ready pool. Variance, skewness, and fourth cumulant (4th) were calculated as averages from 10 traces of the EPSC. They were further time-averaged by a sliding smoothing window of $10 \mathrm{msec}$ in length. The postsynaptic terminal was held at $-80 \mathrm{mV}$. The zero levels in variance, skewness, and fourth moment are shown as broken lines. $B$, The presynaptic terminal was depolarized to $+80 \mathrm{mV}\left(V_{\text {pre }}, 50 \mathrm{msec}\right)$ and then held at $0 \mathrm{mV}$ for $6 \mathrm{msec}$ to evoke a large EPSC. Then the terminal was held at $+20 \mathrm{mV}$ for $100 \mathrm{msec}$ to release the remaining vesicles. The terminal was finally held at $0 \mathrm{mV}$ for $20 \mathrm{msec}$ to confirm that the release-ready pool was depleted. The initial current amplitude (truncated) was $15 \mathrm{nA}$. Traces are arranged as in $A$, but note that the calibrations are different. Variance, skewness, and fourth cumulant $(4 t h)$ were calculated as averages from five EPSC traces.

of the present method to the calyx synapse (Fig. $6 A$ ). A second stimulation protocol was designed to estimate the mEPSC amplitude during an episode of steady-state release after a strong bout of exocytosis (Fig. 6B). Similarly to Neher and Sakaba (2001), we tested these protocols under three conditions: in the absence of CTZ (control condition), in the presence of CTZ (100 $\mu \mathrm{M})$, and in the presence of CTZ plus Kyn (1 mM). Unlike the previous study, the present method does not depend on deconvolution but is based entirely on the analysis of fluctuations.
First, we did a set of experiments under control conditions. We used a voltage-clamp protocol for the presynaptic terminal as shown in Figure $6 A$, which is quite similar to the "early noise" protocol of Neher and Sakaba (2001), except that we aimed at lower release rates. The presynaptic terminal was depolarized from -80 to $+80 \mathrm{mV}$. This activated $\mathrm{Ca}^{2+}$ channels, but the high positive potential primarily prevented $\mathrm{Ca}^{2+}$ influx. Then, the terminal was repolarized to $+55 \mathrm{mV}$ for $100 \mathrm{msec}$. The clamp potential was adjusted so that release rates were low $(<5$ $\mathrm{msec}^{-1}$ ), which is suitable for the analysis described here (Fig. 3, Table 2). During this period, a slowly rising EPSC, recorded at the postsynaptic holding potential of $-80 \mathrm{mV}$, was evoked, which was paralleled by an increase in postsynaptic current noise. Finally, the terminal was held at $0 \mathrm{mV}$ for $20 \mathrm{msec}$ to deplete the releasable pool of synaptic vesicles completely (the depleting pulse), and then the terminal was repolarized to $-80 \mathrm{mV}$. Using 5-10 similar traces (obtained at intervals of 10-15 sec), variance $\left(\lambda_{2}^{\prime}\right)$, skewness $\left(\lambda_{3}^{\prime}\right)$, and fourth cumulant $\left(\lambda_{4}^{\prime}\right)$ were calculated (Fig. $6 A$ ) after mean subtraction and bandpass filtering, as described in Materials and Methods (Eqs. 18-20). To estimate mEPSC amplitudes (and release rates) from skewness and variance, the AMPA channel variance $\left(i^{\prime}\right)$ must be subtracted from the total variance. Measuring $\lambda_{2}^{\prime}$ together with $\lambda_{3}^{\prime}, \lambda_{4}^{\prime}$, and mean current $I_{p}$ allows one to solve Equations 2-4 for $i^{\prime}, h$, and $\xi$ according to Equation 16. We estimated $i^{\prime}$ using the EPSC episode, which was recorded between 100 and $200 \mathrm{msec}$, after the depleting pulse. At this time, release rates should be small, but the residual current component (attributable to delayed clearance of glutamate) can still be observed. The lack of asynchronous release during this period is confirmed by the small amount of skew and fourth cumulant (Fig. 6 $A$ ). In our experiments, the apparent channel size $i^{\prime}$ was between 20 and $30 \mathrm{fA}$, which is similar to the estimate from AMPA-induced current noise (Neher and Sakaba, 2001). It did not differ systematically between control and the various conditions of drugs applied. Measurement of $i^{\prime}$ is not necessary if the analysis is based on the fourth cumulant and skewness.

In the second protocol, a $6 \mathrm{msec}$ depolarizing pulse to $0 \mathrm{mV}$ was applied presynaptically, leading to a short EPSC-like current of $15 \mathrm{nA}$ amplitude (Fig. 6B). This stimulus is strong enough to deplete $50 \%$ of the release-ready pool (Wu and Borst, 1999; Sakaba and Neher, 2001). Subsequently, the terminal was held at $20 \mathrm{mV}$ for $100 \mathrm{msec}$ to slowly deplete the remaining pool. This prolonged depolarization led to a steady postsynaptic inward current of only 300 pA (Fig. $6 B$ ), which indicates that postsynaptic receptors were strongly desensitized during this whole period of release (see below). Finally, the terminal was held at $0 \mathrm{mV}$ for 20 msec at which maximal $I_{\mathrm{Ca}}$ was elicited, to confirm vesicle pool depletion.

Figure $7 A$ shows EPSCs and mEPSC amplitudes estimated from skewness and variance during episodes of moderate release, calculated according to Equation 10 after averaging skewness and variance over $20 \mathrm{msec}$ segments. The mEPSC amplitude was initially approximately $-30 \mathrm{pA}$ and then decreased gradually to $-10 \mathrm{pA}$. This trend was not always seen; in some cell pairs, mEPSC amplitudes were constant throughout episodes of moderate release. The $\mathrm{CV}$ of the amplitude estimate of this cell, which is based on $200 \mathrm{msec}(10 \times 20 \mathrm{msec})$, should be $\sim 0.2$, according to the simulation data of Figure 3; the experimental results confirm this expectation. Figure $7 B$ shows EPSC and mEPSC amplitudes after strong release in the second type of protocol (Fig. 6B), and the mEPSC amplitude was only $10 \mathrm{pA}$ in 
A (moderate release)

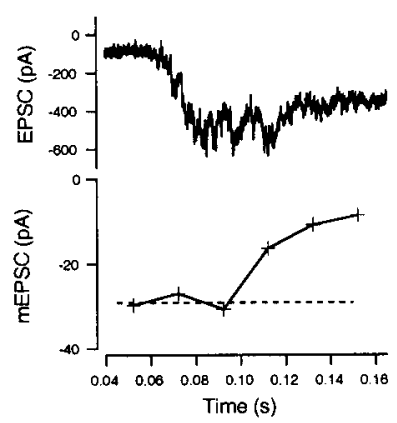

B (after strong release)

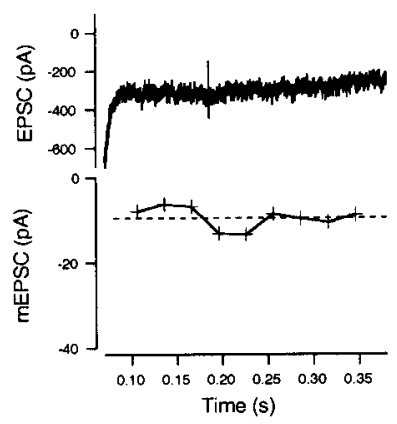

Figure 7. mEPSC amplitudes estimated during periods of moderate release. $A$, An exemplar EPSC trace during moderate stimulation (0.04$0.16 \mathrm{sec}$ in Fig. $6 A$ ) is displayed at expanded scale (top). In the bottom, mEPSCs amplitudes calculated from means of 10 traces of skewness and variance are plotted. The dotted line indicates the mean mEPSC amplitude during the initial periods of moderate release, which is $-29.2 \mathrm{pA}$. $B$, An exemplar EPSC after strong stimulation (from Fig. $6 B ; 0.1-0.35 \mathrm{sec}$ ) is displayed at expanded scale (top). In the bottom, mEPSC amplitudes, calculated from skewness and variance as in $A$, are plotted. The dotted line indicates the mean mEPSC amplitude, which is $-9.5 \mathrm{pA}$.

this case, suggesting desensitization of postsynaptic AMPA receptors.

Second, we performed similar experiments in the presence of $100 \mu \mathrm{M} \mathrm{CTZ} \mathrm{(Figs.} \mathrm{8,} \mathrm{9),} \mathrm{an} \mathrm{agent} \mathrm{known} \mathrm{to} \mathrm{suppress} \mathrm{or} \mathrm{retard}$ desensitization in several glutamatergic synapses (Trussell et al., 1993; Yamada and Tang, 1993). In Figure 8, it is seen that substantial residual currents develop when a large fraction of the releasable pool of vesicles is released. Figure 9 demonstrates that mEPSC estimates are constant under CTZ both during the moderate release episode $(A)$ and after strong release $(B)$.

Third, we estimated mEPSC amplitudes in the presence of CTZ and Kyn (1 mM). Because evoked EPSCs are reduced to $50 \%$ by $1 \mathrm{~mm}$ Kyn, we expected that the underlying mEPSCs should decrease similarly. Because mEPSCs are too small to be resolved directly under this condition (Neher and Sakaba, 2001), this experiment is important for estimating the mEPSC size accurately without any special assumptions. Figure 10 shows an example of recordings in such conditions with a protocol similar to the "early noise" protocol of Figure $6 A$. Variance and skewness (Fig. 10) under Kyn are smaller than those in its absence, consistent with reductions in mEPSC amplitudes. Nevertheless, it was still possible to estimate mEPSC amplitudes from skewness and variance. In the case of Figure 10, the mEPSC amplitude as calculated from Equation 10 was $\sim 13 \mathrm{pA}$. Unfortunately, we were not able to estimate mEPSC amplitudes from the fourth moment and skewness, possibly because the signal of the fourth cumulant is too noisy. It is also difficult to estimate mEPSC amplitudes under CTZ and Kyn, when the residual current is large (data not shown). This is possibly the result of an increased noise level caused by the residual current. Thus, we did no additional measurement under this condition (CTZ plus Kyn).

Figure 11 shows a summary of mEPSC amplitudes estimated from higher moments under three conditions (control, CTZ, and CTZ plus Kyn). Figure $11 A$ compares mEPSC estimates from skewness and variance during moderate release. These were $25.9 \pm 1.7 \mathrm{pA}$ (control; $n=4$ cell pairs), $28.2 \pm 3.6 \mathrm{pA}(\mathrm{CTZ} ; n=$ 6 cell pairs), and $11.8 \pm 2.6 \mathrm{pA}$ (CTZ plus Kyn; $n=5$ cell pairs). Amplitudes under control and CTZ conditions compared well with those of directly sampled mEPSCs. The similarity of mEPSC
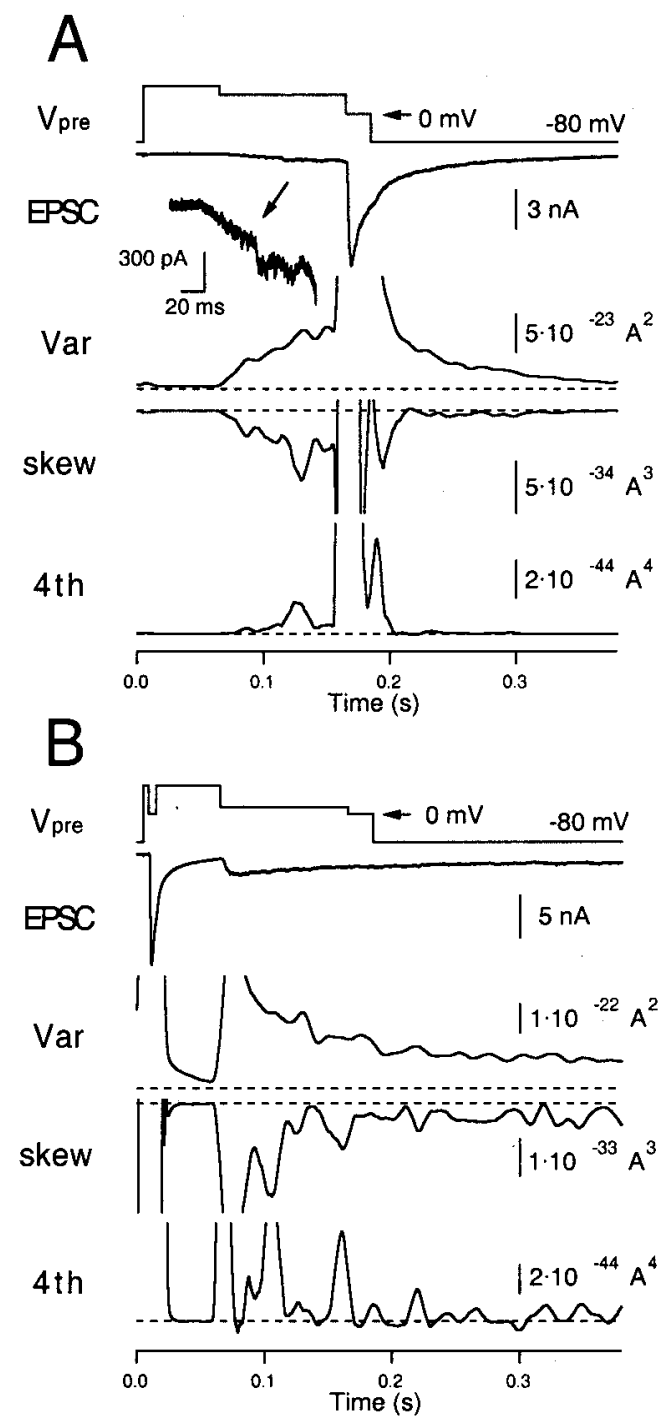

Figure 8. Higher moments measured from an evoked EPSC in the presence of CTZ $(100 \mu \mathrm{M}) . A$, The same protocol as used in Figure $6 A$, except that the extracellular solution contained $100 \mu \mathrm{M}$ CTZ. The early episode, when there is only a small amount of release, is also shown at a larger magnification. $B$, The same protocol as used in Figure $6 B$, except that the extracellular solution contained $100 \mu \mathrm{M}$ CTZ.

amplitudes in the presence and absence of CTZ suggests that postsynaptic receptors did not desensitize significantly during moderate release. Under CTZ plus Kyn, mEPSCs were $42 \%$ of the value obtained in the absence of Kyn. This reduction factor is similar to that of the evoked EPSC $(\sim 50 \%)$ and to the value $(47 \%)$ obtained from an analysis using variance and deconvolution-based release rates (Neher and Sakaba, 2001).

In Figure $11 B$, mEPSC amplitudes estimated during moderate release are compared with those derived from episodes after strong release in the absence of CTZ. In each case, both the estimates from skewness and variance $(3 r d / 2 n d)$ and fourth cumulant and skewness $(4 t h / 3 r d)$ are given. We obtained mEPSC estimates of $25.9 \pm 1.7$ and $35.2 \pm 5.3 \mathrm{pA}(n=4$ cell pairs $)$ when using skewness and variance or fourth cumulant and skewness, respectively. However, the $\mathrm{CV}$ of the fourth cumulant is expected to be quite large (Fig. 3). Therefore, it was necessary to average fourth cumulants over the whole episode of moderate release 
A (moderate release)

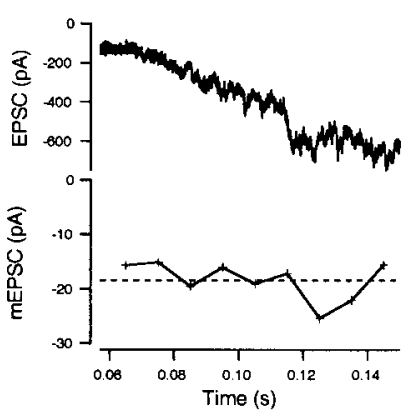

B (after strong release)

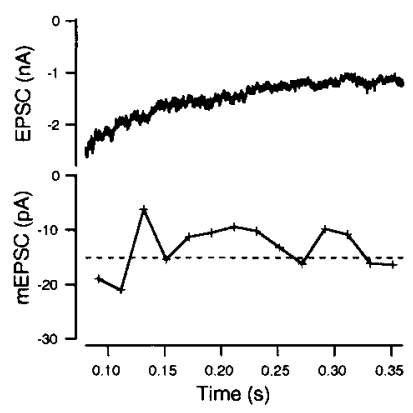

Figure 9. mEPSC amplitudes estimated during periods of moderate release in the presence of CTZ $(100 \mu \mathrm{M})$. A, The same protocol as used in Figure $7 A$, except that the extracellular solution contained $100 \mu \mathrm{M}$ CTZ. $B$, The same protocol as used in Figure $7 B$, except that the extracellular solution contained $100 \mu \mathrm{M} \mathrm{CTZ.}$

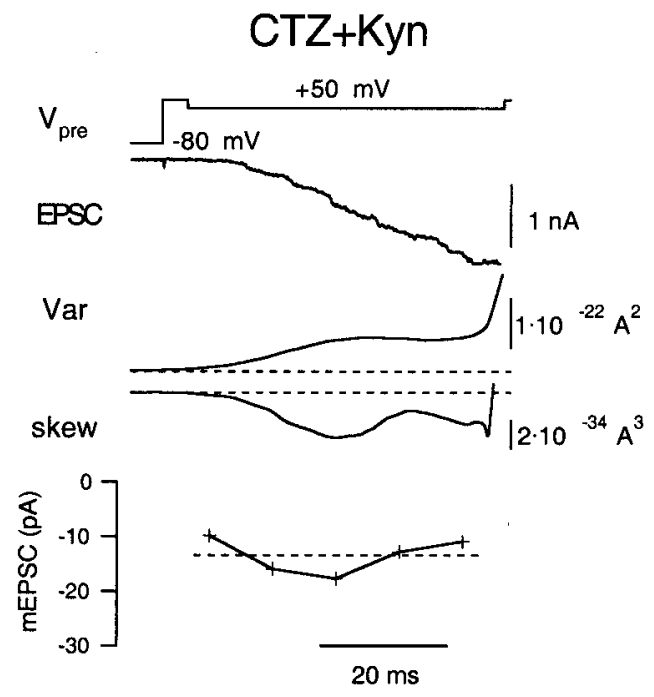

Figure 10. mEPSC amplitudes estimated during moderate release in the presence of CTZ $(100 \mu \mathrm{M})$ plus Kyn $(1 \mathrm{~mm})$. The protocol is similar to that of Figure $6 A$. From top, Presynaptic holding potential $\left(V_{\text {pre }}\right)$, EPSC, variance, skewness, and mEPSC amplitudes estimated from skewness and variance.

( 0.07 to $0.15 \mathrm{sec}$ in Figs. $6 A$ and $7 A$ ) before calculating an mEPSC amplitude estimate. The amplitude estimates based on skew and variance were smaller, whereas those based on the fourth cumulant were larger than the mean of the mEPSC amplitude distribution. On the basis of the simulations, however, we consider the former as more reliable. During steady-state release after an initial bout of strong release, a mean mEPSC amplitude of $9.1 \pm$ $1.3 \mathrm{pA}$ was estimated from skewness and variance. From the fourth cumulant and skewness, a value of $16.9 \pm 1.3 \mathrm{pA}$ was obtained (Fig. 11B). Compared with the amplitude estimate during moderate release, the mEPSC amplitude was depressed to values between 35 and $50 \%$.

Figure $11 C$ shows summary results in the presence of CTZ. Six experiments, in which $i^{\prime}$ and cumulants were calculated, resulted in mean mEPSC size of $28.2 \pm 3.6 \mathrm{pA}$ during moderate release periods. Mean values of $21.6 \pm 4.1 \mathrm{pA}$ were estimated after strong release using skewness and variance for estimation. When the same experiments were analyzed for fourth cumulant and skew-

\section{A (moderate release, $3 \mathrm{rd} / 2 \mathrm{nd}$ )}

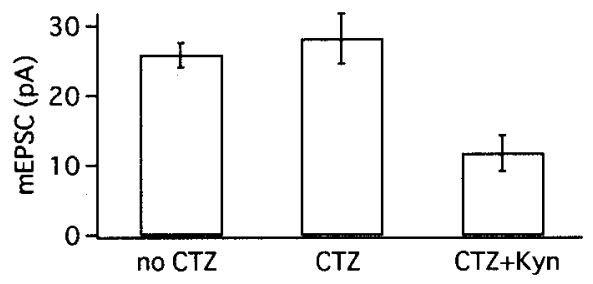

B (no CTZ)

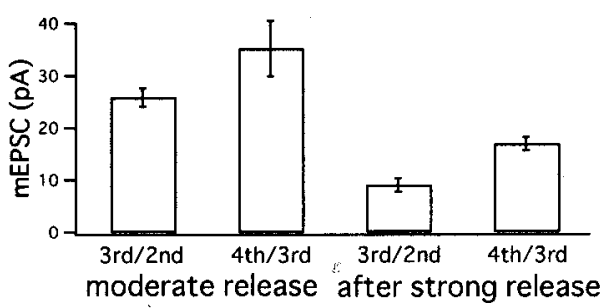

C (CTZ)

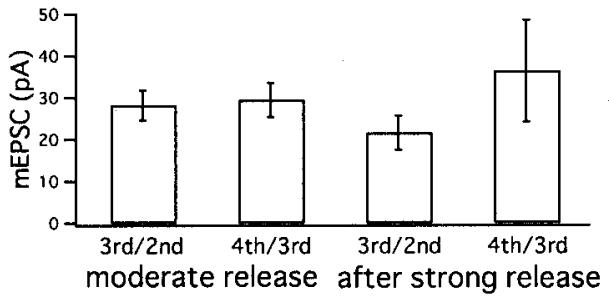

Figure 11. Summary of mEPSC amplitude estimates from higher moments. $A$, mEPSC amplitude estimates during moderate release periods (obtained from protocols as in Fig. $6 A$ ). Values were estimated from skewness and variance. From left to right, mean \pm SEM values of mEPSC obtained in the control condition $(n=4)$, under CTZ $(n=6)$, and under CTZ plus Kyn $(n=5)$. $B$, Mean \pm SEM values of mEPSC amplitude estimates in the absence of CTZ, obtained from four cell pairs. From left to right, Estimates during moderate release periods (according to Figs. $6 A$, $7 A$ ) using skewness and variance, and fourth cumulant and skewness, and then estimates during release periods after strong stimulation (according to Figs. $6 B, 7 B$ ) using skewness and variance, and fourth cumulant and skewness. $C$, The same as $B$, except that experiments were performed in the presence of CTZ. Averages \pm SEM from six cell pairs according to Figures 8 and 9.

ness, we estimated an mEPSC amplitude of $29.5 \pm 4.1 \mathrm{pA}$ for moderate release periods and $36.4 \pm 12.2 \mathrm{pA}$ after strong release. The comparison shows that mEPSC amplitudes are similar or reduced by not more than $20 \%$ after the strong bout of exocytosis in the presence of CTZ. This implies that desensitization of postsynaptic receptors contributes to a decrease in mEPSC amplitudes after strong transmitter release if $\mathrm{CTZ}$ is not present (Meyer et al., 2001; Neher and Sakaba, 2001).

\section{Estimation of release rates using higher moments}

Neher and Sakaba (2001) developed a deconvolution method for calculating transmitter release rates that takes into account the residual current component attributable to delayed clearance of glutamate from the synaptic cleft. By using fluctuation analysis, they showed that release rates can be estimated when desensitization was blocked. However, this method has relatively large errors when release rates are small $\left(<10\right.$ events $\left./ \mathrm{msec}^{-1}\right)$, and the residual current component contributes most of the EPSC. In that case, a small error in the estimation of the residual current causes 
A

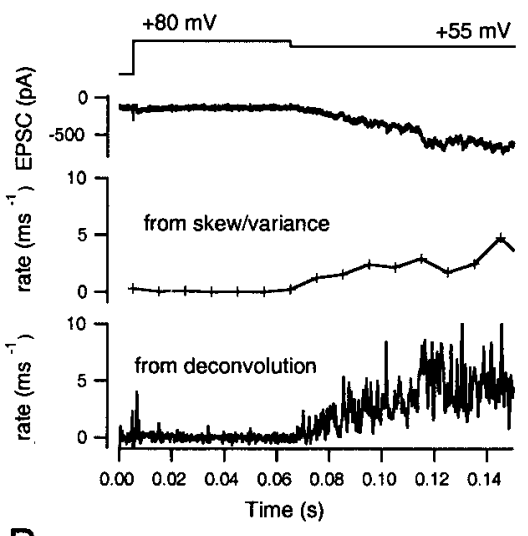

B

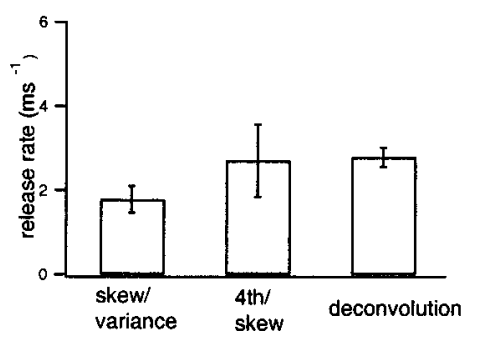

Figure 12. Release rates estimated during episodes of low-frequency release. $A$, The moderate release episode of Figure $8 A$ is displayed at expanded time scale. From top to bottom, Presynaptic holding potential, EPSC, release rate estimated from skewness and variance, and release rate estimated from deconvolution. CTZ $(100 \mu \mathrm{M})$ was added to the extracellular solution. The bottom two traces are based on averages from 10 traces. $B$, Mean \pm SEM values from six cell pairs of measurements, as displayed in $A$. From left to right, Estimates from skewness and variance, from fourth cumulant and skewness, and from deconvolution.

serious overall errors. Furthermore, the method is accurate only when mEPSC amplitude is constant. In contrast, rate estimates using higher moments are reliable when release rates are low (Fig. 3) (Fesce, 1990). In principle, the method is able to simultaneously estimate rate and mEPSC amplitude, although with only limited time resolution. In this section, we therefore estimate release rates using higher moments and compare these with the deconvolution-based rates. For the latter, we assumed an mEPSC amplitude, as given by the mean of its distribution. Experiments were performed in the presence of CTZ, because the constancy of mEPSC amplitude, which is the requirement for deconvolution, can be guaranteed by this drug with an error of $\sim 20 \%$ (Fig. 11) (Neher and Sakaba, 2001). No kynurenic acid was added, however, to preserve large fluctuations.

A first type of experiment was performed to test whether the analysis is reliable under conditions of low release rate (Fig. 12A). Release rates $(\xi)$ were estimated during moderate release periods by holding the presynaptic terminal close to $+55 \mathrm{mV}$. The protocol was the same as has been described in Figure $6 A$. As before, 5-10 similar traces were available form each cell pair. Rate estimates, averaged over $10 \mathrm{msec}$ windows, were well defined, and the time course was similar to that of release rates derived from deconvolution (Fig. 12A). The release rates estimated from skewness and variance usually were slightly smaller than those from the deconvolution (Fig. 12B). To estimate release rates using fourth cumulants and skewness, much more time averaging was required to reduce CVs of these estimates (Fig. 3). Therefore, rates were estimated after averaging fourth cumulants and skew-
A
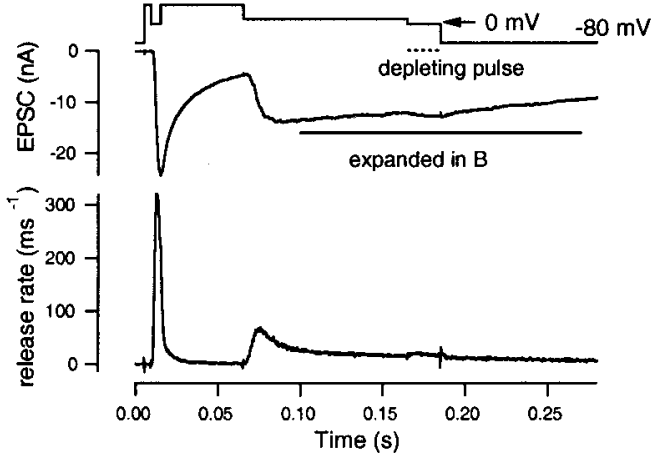

B

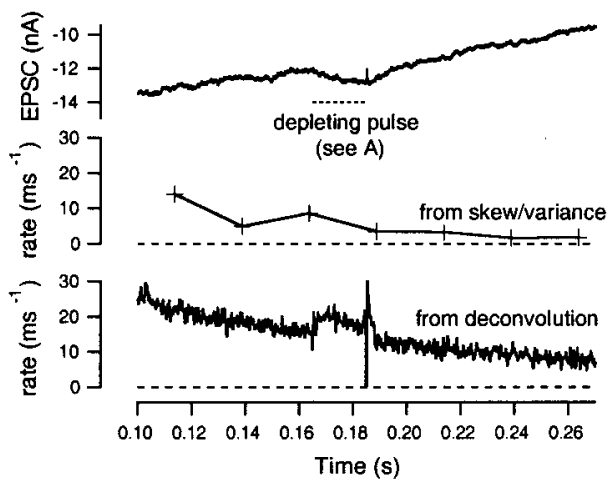

C

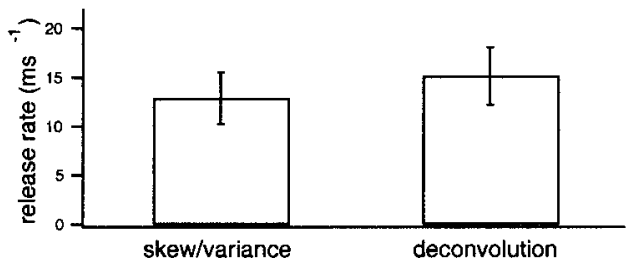

Figure 13. Release rates observed after depletion of the release-ready pool. $A$, The same voltage protocol as used in Figures $6 B$ and $8 B$ was applied to the presynaptic terminal (top trace). The evoked EPSC and the release rate, as estimated from deconvolution, is shown as middle and bottom traces, respectively. CTZ $(100 \mu \mathrm{M})$ was added to the extracellular solution. $B$, Time course of the EPSC observed after depletion of the vesicle pool. The segment from 0.1 to $0.28 \mathrm{sec}$ of $A$ is displayed at expanded scale (top trace), and the release rates, as estimated from skewness and variance (middle trace), and from deconvolution (bottom trace) are shown. The dotted bar indicates the time interval during which $V_{\text {hold }}$ was set to $0 \mathrm{mV}$ for maximum $I_{\mathrm{Ca}} . C$, Mean \pm SEM values of release rates from six cell pairs measured as shown in $B$. Values were estimated from skewness and variance (left) and from deconvolution (right).

ness over the whole period of moderate release (70-130 msec) (Fig. 12B). Averaged over six cell pairs, a mean release rate of $1.8 \pm 0.3$ events/msec was obtained from skewness and variance. This compares with $2.7 \pm 0.9$ events/msec as obtained from fourth cumulant and skewness and with $2.8 \pm 0.2$ events/msec using the deconvolution method (Fig. 12B). Estimates by using higher moments were slightly smaller than those using deconvolution. However, considering that deconvolution is unreliable when release rates are small (as mentioned above), these differences may not be significant.

Next, we performed experiments to see whether consistent estimates for the release rate are obtained under conditions of steady-state release (Fig. 13). We did this under CTZ with strong continuous stimulation, including a short episode of maximum $I_{\mathrm{Ca}}$, which completely depleted the release-ready pool of vesicles, using the protocol as has been described in Figures $6 B$ and $8 B$. 
Release rates under these conditions were low (which was appropriate for estimation using higher moments) despite the strong continuous stimulus, because a large fraction of the release-ready pool had already been released earlier in the record. Low rates of release continued after this depolarization, probably because $\left[\mathrm{Ca}^{2+}\right]_{\mathrm{i}}$ was elevated globally. Release should represent mainly the rapid exocytosis of newly recruited vesicles. Therefore, this measurement also constitutes a test of the concept of the releasable pool and its refilling, as described in the recent work of $\mathrm{Wu}$ and Borst (1999), Schneggenburger et al. (1999), and Sakaba and Neher (2001). The expectation from this concept is that release rates toward the end of a strong stimulus should be approximately similar to that predicted from the time course of recovery from synaptic depression.

We analyzed release rates during continuous depolarization (100 msec) to $+20 \mathrm{mV}$ and the episodes after (0.1-0.28 sec in Fig. $13 A, B)$. As seen from the deconvolution results, release rates were quite low during this period (Fig. 13A,B) and hardly changed when $I_{\mathrm{Ca}}$ was switched to maximum, which is consistent with the notion that the releasable pool of vesicles is depleted. Release rates during this period were estimated using skewness and variance, which showed that rates were on the order of 5-10 $\mathrm{msec}^{-1}$ (Fig. 13B). The rate estimates from skewness and variance were somewhat lower than those from deconvolution in the case of the experiment shown in Figure 13B. However, average values from six cell pairs were similar when both methods were compared (average from 0.1 to $0.25 \mathrm{sec}$ in Fig. $13 A, B)(12.9 \pm 2.6$ events/ msec from skewness and variance and $15.2 \pm 2.9$ events $/ \mathrm{msec}$ from the deconvolution). Estimates from fourth cumulant and skewness showed large fluctuations and were not meaningful, because release rates during this period were higher than those required for a reliable measurement (Fig. 3). From deconvolution, we estimated a mean size of the releasable pool of $3640 \pm$ 692 vesicles (Sakaba and Neher, 2001). Therefore, a release rate of $\sim 10-15$ events/msec, which reflects the recruitment of synaptic vesicles to the releasable pool, would result in a recovery time constant of $300-400 \mathrm{msec}$. This is very similar to the recovery time constant of the pool observed by Wu and Borst (1999) after strong synaptic depression.

\section{DISCUSSION}

Postsynaptic currents fluctuate strongly whenever presynaptic $\left[\mathrm{Ca}^{2+}\right]_{\mathrm{i}}$ rises for periods longer than the duration of a single mEPSC. At glutamatergic synapses, such fluctuations depend on four parameters: the presynaptic transmitter release rate, the size of the postsynaptic quantum (i.e., the amplitude of the mEPSC), the amplitude of the residual current (i.e., the current attributable to glutamate persisting in the synaptic cleft), and the amplitude of the single channel response. Single channel fluctuations contribute to the amplitude dispersion of mEPSCs, and they render the residual current noisy. We provide a theory that allows to calculate the four parameters from the postsynaptic current, its variance, skewness, and the fourth cumulant. For this analysis, postsynaptic records are required, which are either stationary or vary slowly. By analyzing simulated currents, we confirm the expectation (Fesce, 1990) that high-pass filtering of the currents makes the analysis quite immune against nonstationarities, such that valid results could be obtained under a surprisingly wide range of stimulation conditions (Figs. 1-5). Using simulated records, we determine the accuracy of such noise estimates, and we show, for instance, that a $20 \%$ accurate estimate of variance can be obtained from a record as short as $50 \mathrm{msec}$ (Fig. 3, Table 2 ). Finally, we apply the method to records from the calyx of Held synapse and show that accurate estimates for mEPSC amplitudes are obtained (Figs. 6-11). For very strong, longlasting stimulation of the presynaptic terminal, we found steady-state release rates on the order of magnitude of 10 events/msec, well compatible with the rate of recruitment of new vesicles to a completely depleted releasable pool (Fig. 13). Thus, our present studies have two important implications: (1) they provide convenient tools to study low rates of quantal release without complicated assumptions, and (2) they constitute a critical test of the concept of the releasable pool of vesicles at the calyx of Held (Schneggenburger et al., 1999; Wu and Borst, 1999; Sakaba and Neher, 2001).

The method presented here is an extension of previous work on end plate potentials at the neuromuscular junction (Segal et al., 1985; Fesce et al., 1986; Fesce, 1990), allowing one to handle the additional complications attributable to residual current. It has improved time resolution compared with previous studies by taking advantage of optimal filtering to extract relevant information. An essential element in our analysis is high-pass filtering of the postsynaptic currents (Fig. 1) before subjecting them to fluctuation analysis. This has been proposed previously (Segal et al., 1985; Fesce et al., 1986) to be very beneficial for eliminating trends and for improving resolution. We show by simulation that, indeed, the resolution of all of the estimates is far less than optimal when a relatively low high-pass-filtering frequency is used. With the simple filtering tools of our analysis platform (IGOR; WaveMetrics Inc.), a suitable software bandpass filter can be constructed by performing smoothing operations with a smoothing window of $0.3 \mathrm{msec}$, which is approximately $1.5-2$ times longer than the rise time of the mEPSC (see Materials and Methods). The resolution might be improved by choosing a bandpass centered at even higher frequencies. However, then the result is expected to depend critically on clamp speed and on changes in mEPSC rise time, which may result from insufficient clamp.

The method is particularly well suited for low release rates, complementing the recently introduced method of Neher and Sakaba (2001), which combines measurement of variance with deconvolution. The latter method is inaccurate at low release rates (see below); however, it is well suited for measuring rapidly changing release rates under conditions in which the mEPSC amplitude can be expected to be constant. Both of these methods complement the traditional (for review, see McLachlan, 1978; Fesce, 1990; Walmsley, 1993) and more recent forms of variance mean analysis of synaptic transmission (Silver et al., 1998; Clements and Silver, 2000; Oleskevich et al., 2000; Meyer et al., 2001; Scheuss and Neher, 2001). Contrary to these methods, which analyse trial-to-trial fluctuations in highly synchronized (action potential induced) responses, our technique is applicable to slowly changing streams of mEPSCs. It has been difficult to estimate such low release rates at CNS synapses because of the overlapping current components attributable to spillover of released transmitter (Barbour and Häusser, 1997). Whenever such spillover currents can be expected to change slowly with respect to the mEPSC rise time, they should not influence the results of our analysis. Furthermore, the method should not be sensitive to small changes in clamp speed when the low-pass section of the bandpass filter (set by a smoothing time window of $0.3 \mathrm{msec}$ in our study) is selected to be somewhat slower than the slowest 
mEPSC rise time. Therefore, we think that in cases in which the underlying quantal release is relatively homogeneous (and characterized by a relatively well defined time course and amplitude distribution), it should be possible to apply the method to study EPSCs in other synapses. These might be evoked, for example, by focal stimulation (Stevens and Wang, 1995) or by pharmacological manipulations, such as using sucrose and latrotoxin (Rosenmund and Stevens, 1996; Auger and Marty, 1997). Our method should also be useful for analysing asynchronous release (del Castillo and Katz, 1954; Rahamimoff and Yaari, 1973; Goda and Stevens, 1994; Atluri and Regehr, 1998). Especially, the method will be helpful for nonspiking synapses (photoreceptors, Ashmore and Copenhagen, 1980; Schnapf and Copenhagen, 1982; Rieke and Schwartz, 1994, 1996; DeVries and Schwartz, 1999) (retinal bipolar cells, Mittman et al., 1990; von Gersdorff and Matthews, 1994; Lagnado et al., 1996; Matsui et al., 1998) (retinal amacrine cells, Borges et al., 1995) (hair cells, Furukawa et al., 1978; Yamashita and Ohmori, 1990; Parsons et al., 1994; Moser and Beutner, 2000) and GABAergic terminals ( $\mathrm{Lu}$ and Trussell, 2000) in which asynchronous release plays an important role in transmission. Furthermore, the method might give some hints on dissecting membrane potential fluctuations observed at the neuronal soma in which various inputs converge, and such fluctuations are important determinants for action potential timing (Mainen and Sejnowski, 1995; Stevens and Zador, 1998).

When applied to the calyx of Held, the method allows one to obtain an answer to important questions of synaptic physiology, such as on the contribution of postsynaptic factors that determine mEPSC amplitudes and on the validity of the vesicle pool concept.

Regarding the first issue, we have shown previously that strong excitation at the calyx of Held synapse leads to desensitization of postsynaptic receptors (Meyer et al., 2001; Neher and Sakaba, 2001). We confirm this with the new approach based simply on the fluctuations of the signal (Figs. 6, 7). The finding is consistent with results from other calyx-type synapses (Trussell et al., 1993; Otis et al., 1996b; Oleskevich et al., 2000). CTZ partially abolished the reduction in the mEPSC size, but possibly mEPSCs are still reduced by $\sim 20 \%$ (Figs. 8 , 9), which may be attributable to saturation of postsynaptic receptors or slowly developing desensitization (Partin et al., 1994; Geiger et al., 1995). We conclude that the simple use of the amplitude of EPSCs as an index of transmitter release is definitely not a reliable way of estimating quantal release in the absence of CTZ when strong stimuli are applied. There might be less of a problem, however, if one restricts the analysis to low rates of release $\left(<20 \mathrm{msec}^{-1}\right)$ because mEPSCs are not reduced under conditions of mild stimulation (Figs. 6, 8). On the other hand, such a restriction prevents a better understanding of synaptic function, given the fact that changes in EPSC may be attributable to change in release probability or a consequence of changes in vesicle pool size, the latter of which can be only estimated by applying strong stimuli that can deplete the whole vesicle pool (Wu and Borst, 1999).

In many types of synapses, one can define a so-called releasable pool of vesicles consisting of those vesicles that can be released within a very short time $(10-100 \mathrm{msec})$ by a strong stimulus, such as a depolarization to maximum $I_{\mathrm{Ca}}$ (Wu and Borst, 1999; Sakaba and Neher, 2001), a volley of action potentials (Dobrunz and Stevens, 1997; Schneggenburger et al., 1999), or a hyperosmotic stimulus (Rosenmund and Stevens, 1996). The size of this pool is usually calculated from some measure of the cumulative response divided by the unit response. This approach assumes the unit response to be constant. It has been shown, however, that mEPSC amplitudes decrease during strong stimulation because of receptor desensitization (Trussell et al., 1993; Otis et al., 1996b). This can lead to erroneous estimates of pool size (Sakaba and Neher, 2001). Indeed, no proof has been available so far that the final decay of the postsynaptic current in response to a pool-depleting stimulus is not primarily attributable to desensitization. In that case, the pool concept would be invalidated. Neher and Sakaba (2001) showed, by combining variance analysis with deconvolution, that, under CTZ, the mEPSC size stays relatively constant up to $\sim 80 \%$ depletion of the releasable pool. However, the method used in this previous investigation is not very accurate at low release rates and when a large residual current is present, two conditions that become more and more serious as the pool depletes.

The method that we present here is optimally suited for low release rates, as are measured after complete pool depletion. We applied it to this condition under CTZ and, indeed, found a release rate of $\sim 10$ events/msec (Fig. 13). This rate most likely reflects the refilling rate of synaptic vesicles to the releasable pool, the value being consistent with the pool recovery time course estimated from dual-pulse experiment by Wu and Borst (1999). This study relied on the assumption of constant mEPSCs during the stimulus, which could not be proven by the method used. Furthermore, the time course of recovery from depression may be complicated by contributions of mechanisms other than a depletion-refilling process (Hsu et al., 1996; Dobrunz et al., 1997; Bellingham and Walmsley, 1999). Nevertheless, the agreement between the recovery time constant by Wu and Borst (1999) and the refilling rates demonstrated here favors a simple mechanism of pool depletion and refilling.

We also showed that mEPSC size is nearly constant after full vesicle depletion (Fig. 11). This indicates that vesicles only partially filled with transmitter cannot contribute substantially to the EPSC, which might be expected if vesicles recycle rapidly during strong stimulation (Pyle et al., 2000). In addition, a relatively normal mEPSC size even after strong stimulation suggests that not many synaptic vesicles fuse at locations remote from postsynaptic densities; one might expect this to happen if globally elevated $\mathrm{Ca}^{2+}$ starts to trigger release of vesicles in parts of the terminal, which are remote from postsynaptic densities.

In summary, we provided the theoretical basis and demonstrated experimental usefulness of higher moments of current fluctuations for the analysis of transmitter release. We hope this method to be helpful for a detailed study of synaptic function in various synapses.

\section{REFERENCES}

Anderson CR, Stevens CF (1973) Voltage clamp analysis of acetylcholine produced end-plate current fluctuations at frog neuromuscular junction. J Physiol (Lond) 235:655-691.

Ashmore JF, Copenhagen DR (1980) Different postsynaptic events in two types of retinal bipolar cells. Nature 288:84-86.

Atluri PP, Regehr WG (1998) Delayed release of neurotransmitter release from cerebellar granule cells. J Neurosci 18:8214-8227.

Auger C, Marty A (1997) Heterogeneity of functional synaptic parameters among single release sites. Neuron 19:139-150.

Barbour B, Häusser M (1997) Intersynaptic diffusion of neurotransmitter. Trends Neurosci 20:377-384.

Barbour B, Keller BU, Llano I, Marty A (1994) Prolonged presence of 
glutamate during excitatory synaptic transmission to cerebellar Purkinje cells. Neuron 12:1331-1343.

Bellingham MC, Walmsley B (1999) A novel presynaptic inhibitory mechanism underlies paired pulse depression at a fast central synapse. Neuron 23:159-170.

Borges S, Gleason E, Turelli M, Wilson M (1995) The kinetics of quantal transmitter release from retinal amacrine cells. Proc Natl Acad Sci USA 92:6896-6900.

Borst JGG, Sakmann B (1996) Calcium influx and transmitter release in a fast CNS synapse. Nature 383:431-434.

Campbell N (1909) The study of discontinuous phenomena. Proc Camb Philol Soc 15:117-136.

Carter AG, Regehr WG (2000) Prolonged synaptic currents and glutamate spillover at the parallel fiber to stellate cell synapse. J Neurosci 20:4423-4434

Chuhma N, Ohmori H (1998) Postnatal development of phase-locked high-fidelity synaptic transmission in the medial nucleus of the trapezoid body of the rat. J Neurosci 18:512-520.

Clements JD, Silver RA (2000) Unveiling synaptic plasticity: a new graphical and analytical approach. Trends Neurosci 23:105-113.

Clements JD, Lester RAJ, Tong G, Jahr CE, Westbrook GL (1992) The time course of glutamate in the synaptic cleft. Science 258:1498-1501.

Colquhoun D, Hawkes AG (1981) On the stochastic properties of single ion channels. Proc R Soc Lond B Biol Sci 211:205-235.

Courtney KR (1978) Extended moment analysis for binomial parameters of transmitter release. J Theor Biol 73:285-292.

Crank J (1975) The mathematics of diff usion. Oxford, UK: Clarendon.

del Castillo J, Katz B (1954) Statistical factors involved in neurotransmitter facilitation and depression. J Physiol (Lond) 124:574-585.

DeVries SH, Schwartz EA (1999) Kainate receptors mediate synaptic transmission between cones and "Off" bipolar cells in a mammalian retina. Nature 397:157-160.

Dobrunz LE, Stevens CF (1997) Heterogeneity of release probability, facilitation, and depletion at central synapses. Neuron 18:995-1008.

Dobrunz LE, Huang EP, Stevens CF (1997) Very short-term plasticity in hippocampal synapses. Proc Natl Acad Sci USA 94:14843-14847.

Fesce R (1990) Stochastic approaches to the study of synaptic function. Prog Neurobiol 35:85-133

Fesce R, Segal JR, Hurlbut WP (1986) Fluctuation analysis of nonideal shot noise. Application to the neuromuscular junction. J Gen Physiol $88: 25-57$.

Furukawa T, Hayashida Y, Matsuura S (1978) Quantal analysis of the size of excitatory post-synaptic potentials at synapses between hair cells and afferent nerve fibers in goldfish. J Physiol (Lond) 276:211-226.

Geiger JR, Melcher T, Koh DS, Sakmann B, Seeburg PH, Jonas P, Monyer H (1995) Relative abundance of subunit mRNAs determines gating and $\mathrm{Ca}^{2+}$ permeability of AMPA receptors in principal neurons and interneurons in rat CNS. Neuron 15:193-204.

Goda Y, Stevens CF (1994) Two components of transmitter release at a central synapse. Proc Natl Acad Sci USA 91:12942-12946.

Heinemann SH, Sigworth FJ (1991) Open channel noise. VI. Analysis of amplitude histograms to determine rapid kinetic parameters. Biophys J 60:577-587.

Heinemann SH, Sigworth FJ (1993) Fluctuations of ionic currents and ion channel proteins, $\mathrm{Ch}$ 12. In: Thermodynamics of membrane receptors and channels (Jackson MB, ed), pp 407-422. Boca Raton, FL: CRC.

Hsu SF, Augustine GJ, Jackson MB (1996) Adaptation of $\mathrm{Ca}^{2+}$. triggered exocytosis in presynaptic terminals. Neuron 17:501-512.

Jonas P, Major G, Sakmann B (1993) Quantal components of unitary EPSCs at the mossy fibre synapse on CA3 pyramidal cells of rat hippocampus. J Physiol (Lond) 472:615-663.

Katz B, Miledi R (1972) The statistical nature of the acetylcholine potential and its molecular components. J Physiol (Lond) 224:665-700

Kinney GA, Overstreet LS, Slater NT (1997) Prolonged physiological entrapment of glutamate in the synaptic cleft of cerebellar unipolar brush cells. J Neurophysiol 78:1320-1333.

Lagnado L, Gomis A, Job C (1996) Continuous vesicle cycling in the synaptic terminal of retinal bipolar cells. Neuron 17:957-967.

Liu G, Choi S, Tsien RW (1999) Variability of neurotransmitter concentration and nonsaturation of postsynaptic AMPA receptors at synapses in hippocampal cultures and slices. Neuron 22:395-409.

Lu T, Trussell LO (2000) Inhibitory transmission mediated by asynchronous transmitter release. Neuron 26:683-694.

Mainen ZF, Sejnowski TJ (1995) Reliability of spike timing in neocortical neurons. Science 268:1503-1506.

Martin C, Finger W (1998) Veratridine-induced high-frequency asynchronous release of inhibitory transmitter quanta in crayfish nervemuscle synapses superfused with normal and low-calcium saline. Pflügers Arch 411:469-477.

Matsui K, Hosoi N, Tachibana M (1998) Excitatory synaptic transmission in the inner retina: paired recordings of bipolar cells and neurons of the ganglion cell layer. J Neurosci 18:4500-4510.

McLachlan EM (1978) The statistics of transmitter release at chemical synapses. International review of physiology, Vol 17, Neurophysiology III (Porter R, ed), pp 49-117, Baltimore: University Park.

Mennerick S, Zorumski CF (1995) Presynaptic influence on the time course of fast excitatory synaptic currents in cultured hippocampal cells. J Neurosci 15:3178-3192.

Meyer A, Neher E, Schneggenburger R (2001) Estimation of quantal size and number of functional active zones at the calyx of Held synapse by non-stationary EPSC variance analysis. J Neurosci 21:7889-7900.

Mittman S, Taylor WR, Copenhagen DR (1990) Concomitant activation of two types of glutamate receptor mediates excitation of salamander retinal ganglion cells. J Physiol (Lond) 428:175-197.

Moser T, Beutner D (2000) Kinetics of exocytosis and endocytosis at the cochlear inner hair cell afferent synapse of the mouse. Proc Natl Acad Sci USA 97:883-888.

Neher E, Sakaba T (2001) Combining deconvolution and noise analysis for the estimation of transmitter release rates at the calyx of Held. J Neurosci 21:444-461.

Neher E, Stevens CF (1977) Conductance fluctuations and ionic pores in membranes. Annu Rev Biophys Bioeng 6:345-381.

Oleskevich S, Celements J, Walmsley B (2000) Release probability modulates short-term plasticity at a giant terminal. J Physiol (Lond) 524:512-523.

Otis TS, Wu YC, Trussell LO (1996a) Delayed clearance of transmitter and the role of glutamate transporters at synapses with multiple release sites. J Neurosci 16:1634-1644.

Otis TS, Zhang S, Trussell LO (1996b) Direct measurement of AMPA receptor desensitization induced by glutamatergic synaptic transmission. J Neurosci 16:7496-7504.

Parsons TD, Lenzi D, Almers W, Roberts WM (1994) Calciumtriggered exocytosis and endocytosis in an isolated presynaptic cell: capacitance measurements in saccular hair cells. Neuron 13:875-883.

Partin KM, Patneau DK, Mayer ML (1994) Cyclothiazide differentially modulates desensitization of alpha-amino-3-hydroxy-5-methyl-4isoxazolepropionic receptor splice variants. Mol Pharmacol 46:129-138.

Partin KM, Fleck MW, Mayer ML (1996) AMPA receptor flip/flop mutants affecting deactivation, desensitization, and modulation by cyclothiazide, aniracetam, and thiocyanate. J Neurosci 16:6634-6647.

Pyle JL, Kavalali ET, Piedras-Renteria ES, Tsien RW (2000) Rapid reuse of readily releasable vesicles at hippocampal synapses. Neuron 28:221-231.

Rahamimoff R, Yaari Y (1973) Delayed release of transmitter at the frog neuromuscular junction. J Physiol (Lond) 228:241-257.

Rice SO (1944) Mathematical analysis of random noise. Bell Tel Sys J 23:282-332.

Rieke F, Schwartz EA (1994) A cGMP-gated current can control exocytosis at cone synapses. Neuron 13:863-873.

Rieke F, Schwartz EA (1996) Asynchronous transmitter release: control of exocytosis and endocytosis at the salamander rod synapse. J Physiol (Lond) 493:1-8.

Rosenmund C, Stevens CF (1996) Definition of the readily releasable pool of vesicles at hippocampal synapses. Neuron 16:1197-1207.

Rosenmund C, Stern-Bach Y, Stevens CF (1998) The tetrameric structure of a glutamate receptor channel. Science 280:1596-1599.

Rossi ML, Martini M, Pelucchi B, Fesce R (1994) Quantal nature of synaptic transmission at the cytoneural junction in the frog. J Physiol (Lond) 478:17-35

Sakaba T, Neher E (2001) Quantitative relationship between transmitter release and calcium current at the calyx of Held synapse. J Neurosci 21:462-476.

Scheuss V, Neher E (2001) Estimating synaptic parameters from mean, variance and covariance in trains of synaptic responses. Biophys $\mathrm{J}$ 81:1970-1989.

Schnapf JL, Copenhagen DR (1982) Differences in the kinetics of rod and cone synaptic transmission. Nature 296:862-864.

Schneggenburger R, Meyer AC, Neher E (1999) Released fraction and total size of a pool of immediately available transmitter quanta at a calyx synapse. Neuron 23:399-409.

Segal JR, Ceccarelli B, Fesce R, Hurlbut WP (1985) Miniature endplate potential frequency and amplitude determined by an extension of Campbell's theorem. Biophys J 47:183-202.

Sigworth F (1980) The variance of sodium current fluctuations at the node of Ranvier. J Physiol (Lond) 307:97-129.

Silver RA, Momiyama A, Cull-Candy SG (1998) Locus of frequencydependent depression identified with multiple-probability fluctuation analysis at rat climbing fibre-Purkinje cell synapses. J Physiol (Lond) 518:121-130.

Stevens CF, Wang Y (1995) Facilitation and depression at single central synapses. Neuron 14:795-802.

Stevens CF, Zador AM (1998) Input synchrony and the irregular firing of cortical neurons. Nat Neurosci 1:210-217.

Tang CM, Margulis M, Shi QY, Fielding A (1994) Saturation of postsynaptic glutamate receptors after quantal release of transmitter. Neuron 13:1385-1393. 
Traynelis SF (1998) Software-based correction of single compartment series resistance errors. J Neurosci Methods 86:25-34.

Traynelis SF, Jaramillo F (1998) Getting the most out of noise in the central nervous system. Trends Neurosci 21:137-145.

Trussell LO, Zhang S, Raman IM (1993) Desensitization of AMPA receptors upon multiquantal neurotransmitter release. Neuron 10:1185-1196.

von Gersdorff, Matthews G (1994) Dynamics of synaptic vesicle fusion and membrane retrieval in synaptic terminals. Nature 367:735-739.

Walmsley B (1993) Quantal analysis of synaptic transmission. In: Electrophysiology, a practical approach (Wallis DI, ed), pp 109-141. Oxford: IRL.

Wu LG, Borst JGG (1999) The reduced release probability of releasable vesicles during recovery from short-term synaptic depression. Neuron 23:821-832.

Yamada KA, Tang CM (1993) Benzothiazides inhibit rapid glutamate receptor desensitization and enhance glutamatergic synaptic currents. J Neurosci 13:3904-3915.

Yamashita M, Ohmori H (1990) Synaptic responses to mechanical stimulation in calyceal and bouton type vestibular afferents studied in an isolated preparation of semicircular canal ampullae of chicken. Exp Brain Res 80:475-488

Zucker RS, Kullmann DM, Bennett M (1999) Release of neurotransmitters, Ch 7. In: Fundamental neuroscience. II. Cellular and molecular neuroscience (Zigmond MJ, Bloom FE, Landis S, Roberts JL, Squire LR, eds), pp 155-192. New York: Academic. 